\title{
Hybrid Monte Carlo on Lefschetz Thimbles - A study of the residual sign problem -
}

\author{
H. Fujii, D. Honda, M. Kato, Y. Kikukawa, S. Komatsu and T. Sano ${ }^{b}$ \\ Institute of Physics, the University of Tokyo, Tokyo 153-8902, Japan \\ ${ }^{b}$ Theoretical Research Division, RIKEN Nishina Center, Wako 2-1, Saitama 351-0198, Japan \\ E-mail: hfujii@phys.c.u-tokyo.ac.jp, dhonda@hep1.c.u-tokyo.ac.jp, \\ kato@hep1.c.u-tokyo.ac.jp, kikukawa@hep1.c.u-tokyo.ac.jp, \\ skomatsu@hep1.c.u-tokyo.ac.jp, tsano@riken.jp
}

Abstract: We consider a hybrid Monte Carlo algorithm which is applicable to lattice theories defined on Lefschetz thimbles. In the algorithm, any point (field configuration) on a thimble is parametrized uniquely by the flow-direction and the flow-time defined at a certain asymptotic region close to the critical point, and it is generated by solving the gradient flow equation downward. The associated complete set of tangent vectors is also generated in the same manner. Molecular dynamics is then formulated as a constrained dynamical system, where the equations of motion with Lagrange multipliers are solved by the second-order constraint-preserving symmetric integrator. The algorithm is tested in the $\lambda \phi^{4}$ model at finite density, by choosing the thimbles associated with the classical vacua for subcritical and supercritical values of chemical potential. For the lattice size $L=4$, we find that the residual sign factors average to not less than 0.99 and are safely included by reweighting and that the results of the number density are consistent with those obtained by the complex Langevin simulations.

Keywords: Lattice Field Theory, Monte Carlo simulation, Lefschetz thimble 


\section{Contents}

1 Introduction 1

2 Complexified models on Lefschetz thimbles 3

3 An algorithm of hybrid Monte Carlo on Lefschetz thimbles $\quad 7$

$\begin{array}{lll}3.1 & \text { To generate a thimble by solving the flow equations downward } & 7\end{array}$

$\begin{array}{llr}3.2 & \text { Constrained molecular dynamics } & 8\end{array}$

$\begin{array}{ll}3.3 \text { Hybrid Monte Carlo updates } & 10\end{array}$

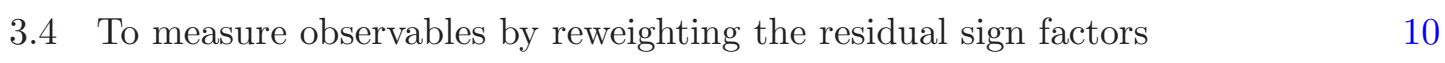

4 HMC simulations of the complexified $\lambda \phi^{4}$ model at finite density $\quad 11$

$\begin{array}{lll}4.1 & \text { Thimble 1-(a) for } \mu<\tilde{\mu}_{c} & 13\end{array}$

$\begin{array}{lll}4.2 & \text { Thimble 2-(b) for } \mu>\tilde{\mu}_{c} & 15\end{array}$

$\begin{array}{ll}\text { 4.3 A comparison to the results of the complex Langevin simulations } & 18\end{array}$

5 Summary and Discussion $\quad 21$

A Tangent vectors at the critical point of the thimble 2-(b) 22

\section{Introduction}

Formulated in physically well-reasoned and well-defined manners, several field theories have complex actions in Euclidean lattice. These include QCD at finite density, chiral gauge theories, chiral Yukawa theories, etc. To these theories, the state-of-art Monte Carlo methods do not apply straightforwardly. If there exists a stochastic method which is based on a sound theoretical basis and is applicable to such theories with complex actions, it would allow us to do thorough non-perturbative studies of these theories. ${ }^{1}$

One possible approach to this problem is to consider the field variables, which are assumed to be real in the original formulation, to be complex and to extend the cycle of path-integration to a complex region in order to achieve better convergence. First example is to use complexified Langevin equation[7-9]. Second example is to select Lefschetz thimbles as the cycle of path-integration, where the imaginary part of the complex action stays constant $[10,11]$.

\footnotetext{
${ }^{1}$ In particular, the study of lattice QCD at finite temperature and density, to figure out the phase structure of QCD, is the subject of great interest and there are a lot of research activities. The authors refer the reader to the talks at the International Workshop on the Sign Problem in QCD and Beyond (Regensburg, 2012)[http://www.physik.uni-regensburg.de/sign2012/talks.shtml], and [1-6] for recent reviews and references.
} 
The first method, to use complexified Langevin equation[7-9], is simple in its implementations, but it is not fully understood theoretically in its convergence properties[12-31]. ${ }^{2}$ The recent numerical results by Aarts about the complex $\lambda \phi^{4}$ model $[17]^{3}$ and by Sexty on full QCD [29] are remarkable and encouraging, though.

The second method, to select Lefschetz thimbles as the cycle of path-integration[10, $11,35,36]$, seems generic, but it is not easy in general to know the set of thimbles which is equivalent to the original cycle. Moreover, the path-integration measure in the complexified field space gives rise to an extra complex phase, and to compute the residual phase factor it is required to know the tangent spaces of the thimbles. Recently, AuroraScience collaboration[11] has considered to define a lattice model by the single thimble associated with the Gaussian critical point (or the classical vacuum) and proposed a Langevin simulation algorithm for such models. ${ }^{4}$ The collaboration has then reported a numerical result about the $\lambda \phi^{4}$ model [36], which is consistent with the results obtained by the complex Langevin equation[17] and the dual variable method[32-34]..$^{5}$ In these works, however, the residual phase factor is ignored, and the residual sign problem in the $\lambda \phi^{4}$ model remains to be studied systematically. Quite recently, Mukherjee, Cristoforetti and Scorzato have studied the residual sign problem in the $\mathrm{U}(1)$ one-plaquette model through a new Metropolis sampling method[37]. ${ }^{6}$

The purpose of this article is to introduce a hybrid Monte Carlo algorithm which is applicable to the lattice models defined on Lefschetz thimbles. In this algorithm, any point (field configuration) on a given thimble is parametrized uniquely by the flow-direction and the flow-time defined in a certain asymptotic region close to the critical point, and it is generated by solving the gradient flow equation downward. The complete set of tangent vectors at the point (associated with the field configuration) is also generated, although it is numerically very demanding, but because it is required for the computation of the residual phase factor. Molecular dynamics is then formulated as a constrained dynamical system, where the equations of motion with Lagrange multipliers are solved by the second-order constraint-preserving symmetric integrator[38]. We hope this algorithm can be used for a systematic study of the residual sign problem and other aspects of this second method.

We test the algorithm in the complex $\lambda \phi^{4}$ model at finite density by observing the number density for various values of chemical potential $\mu$. We examine both thimbles associated with the classical vacua for subcritical and supercritical values of $\mu$. For the lattice size $L=4$, we find that the residual sign factors average to not less than 0.99 and

\footnotetext{
${ }^{2}$ See $[5,27]$ for reviews on this approach.

${ }^{3}$ This model has also been simulated successfully by the dual variable method in [32-34].

${ }^{4}$ It has also been shown by the authors of [11] that in such models the symmetry property is preserved and the perturbation theory reproduces the same result as the original one.

${ }^{5}$ Strictly speaking, the field configurations obtained in this simulation do not belong to the Lefschetz thimble associated with the Gaussian critical point (or the classical vacuum). Rather, they are obtained by projecting on to the tangent space at the critical point, although the tangent space is not necessarily in the same homology class as the thimble. Accordingly, the imaginary part of the action does not stay constant, and its exponent is included by reweighting. It has been claimed that this approximation is good enough to reproduce the silver blaze behavior in the $\lambda \phi^{4}$ model.

${ }^{6}$ This Metropolis sampling method is based on a mapping between a thimble and its asymptotic "Gaussian" region close to the critical point.
} 
are safely included by reweighting, and that the results of the number density agree with those obtained by the complex Langevin simulations within statistical errors, except for a few values of $\mu$, and overall, they are consistent with each other.

This paper is organized as follows. In section 2, we review the basics of the complexification of lattice models on Lefschetz thimbles. Section 3 is devoted to the description of the hybrid Monte Carlo algorithm which is applicable to lattice models defined on Lefschetz thimbles. In section 4 , the algorithm is applied to the $\lambda \phi^{4}$ model with chemical potential. In the final section 5 , we conclude with a few discussions.

\section{Complexified models on Lefschetz thimbles}

First we review the basics of the complexification of lattice models on Lefschetz thimbles[10, 11]. Let us consider a lattice theory with $n$ real degrees of freedom and denote the real field variables as $x=\left(x_{1}, \cdots, x_{n}\right)$. It is assumed that $x$ takes the value in a subset $\mathcal{C}_{\mathbb{R}}$ of $\mathbb{R}^{n}$ and the action of the model $S[x]$ has a non-zero imaginary part. The partition function of the model is defined by the path-integration over $\mathcal{C}_{\mathbb{R}}\left(\subseteq \mathbb{R}^{n}\right)$,

$$
Z=\int_{\mathcal{C}_{\mathbb{R}}} \mathcal{D}[x] \exp \{-S[x]\}
$$

where the measure is given by $\mathcal{D}[x]=d^{n} x$.

In complexification, the field variables are extended to complex variables $z \in \mathbb{C}^{n}$, and the action is extended to a holomorphic function of $z, S[z]$. As for the cycle of the path-integration, Morse theory tells us how to select the set of Lefschetz thimbles which is homologically equivalent to $\mathcal{C}_{\mathbb{R}}$. Morse function in our case is defined by $h \equiv-\operatorname{Re} S[z]$ and the associate gradient (downward) flow equation is given by $^{7}$

$$
\frac{d}{d t} z_{i}(t)=\frac{\partial \bar{S}[\bar{z}]}{\partial \bar{z}_{i}} \quad(t \in \mathbb{R})
$$

The set of critical points $\Sigma$ consists of the points $\left\{z_{\sigma}\right\}$ which satisfy $\partial S[z] /\left.\partial \bar{z}_{i}\right|_{z=z_{\sigma}}=0$. Associated with a critical point $z_{\sigma}$, a Lefschetz thimble $\mathcal{J}_{\sigma}$ is defined by the union of all downward flows which trace back to $z_{\sigma}$ at $t=-\infty$. The thimble is a $n$-dimensional real submanifold in $\mathbb{C}^{n}$. One can introduce another $n$-dimensional real submanifold $\mathcal{K}_{\sigma}$ of $\mathbb{C}^{n}$ by the union of all downward flows which converge to $z_{\sigma}$ at $t=+\infty$ so that its intersection number is unity with $\mathcal{J}_{\sigma}$ and vanishing otherwise, $\left\langle\mathcal{J}_{\sigma}, \mathcal{K}_{\tau}\right\rangle=\delta_{\sigma \tau}$. Then, according to Morse theory, it follows that

$$
\mathcal{C}_{\mathbb{R}}=\sum_{\sigma \in \Sigma} n_{\sigma} \mathcal{J}_{\sigma}, \quad n_{\sigma}=\left\langle\mathcal{C}_{\mathbb{R}}, \mathcal{K}_{\sigma}\right\rangle
$$

\footnotetext{
${ }^{7}$ Along the flow, $h$ is monotonically decreasing,

$$
\frac{d}{d t} h=-\frac{1}{2}\left\{\frac{\partial S[z]}{\partial z} \cdot \frac{d}{d t} z(t)+\frac{\partial \bar{S}[\bar{z}]}{\partial \bar{z}} \cdot \frac{d}{d t} \bar{z}(t)\right\}=-\left|\frac{\partial S[z]}{\partial z}\right|^{2} \leq 0,
$$
}

while the imaginary part of the action stays constant,

$$
\frac{d}{d t} \operatorname{Im} S[z]=\frac{1}{2 i}\left\{\frac{\partial S[z]}{\partial z} \cdot \frac{d}{d t} z(t)-\frac{\partial \bar{S}[\bar{z}]}{\partial \bar{z}} \cdot \frac{d}{d t} \bar{z}(t)\right\}=0 .
$$


And the partition function of the model is given by the formula,

$$
\begin{aligned}
Z & =\sum_{\sigma \in \Sigma} n_{\sigma} \exp \left\{-S\left[z_{\sigma}\right]\right\} Z_{\sigma} \\
Z_{\sigma} & =\int_{\mathcal{J}_{\sigma}} \mathcal{D}[z] \exp \left\{-\operatorname{Re}\left(S[z]-S\left[z_{\sigma}\right]\right)\right\} .
\end{aligned}
$$

In this result, for the critical points $\left\{z_{\sigma}\right\}$ satisfying $-\operatorname{Re} S\left[z_{\sigma}\right]>\max \{-\operatorname{Re} S[x]\}\left(x \in \mathcal{C}_{\mathbb{R}}\right)$, it holds that $\left\langle\mathcal{C}_{\mathbb{R}}, \mathcal{K}_{\sigma}\right\rangle=0$ and the associated thimbles do not contribute to the pathintegration. On the other hand, for the critical points $\left\{z_{\sigma}\right\}$ in the original cycle $\mathcal{C}_{\mathbb{R}}$ (i.e. classical solutions in the original theory), it holds that $\left\langle\mathcal{C}_{\mathbb{R}}, \mathcal{K}_{\sigma}\right\rangle=1$ and the associated thimbles contribute with the relative weights proportional to $\exp \left(-S\left[z_{\sigma}\right]\right)$. In particular, for the classical vacuum in the original theory $z_{\text {vac }} \in \mathcal{C}_{\mathbb{R}}$, it holds that $-\operatorname{Re} S\left[z_{\text {vac }}\right]=$ $\max \{-\operatorname{Re} S[x]\}\left(x \in \mathcal{C}_{\mathbb{R}}\right)$ and therefore the associated thimble $\mathcal{J}_{\text {vac }}$ contributes most among all the thimbles. And, in the above formula eq. (2.5), the measure on the thimbles $\mathcal{D}[z]=\left.d^{n} z\right|_{\mathcal{J}_{\sigma}}$ should be specified based on the knowledge of the geometry of $\left\{\mathcal{J}_{\sigma}\right\}$, in particular, their tangent spaces.

As to the expectation value of an observable $O[z]$, it is defined by the formula,

$$
\langle O[z]\rangle=\frac{1}{Z} \sum_{\sigma \in \Sigma} n_{\sigma} \exp \left\{-S\left[z_{\sigma}\right]\right\} Z_{\sigma}\langle O[z]\rangle_{\mathcal{J}}
$$

where

$$
\langle O[z]\rangle_{\mathcal{J}_{\sigma}}=\frac{1}{Z_{\sigma}} \int_{\mathcal{J}_{\sigma}} \mathcal{D}[z] \exp \left\{-\operatorname{Re}\left(S[z]-S\left[z_{\sigma}\right]\right)\right\} O[z]
$$

As a possible and practical approximation to the formula eq. (2.6), one may take the single contribution of the thimble associated with the classical vacuum, $\mathcal{J}_{\text {vac }}$, as considered by AuroraScience collaboration[11]. ${ }^{8}$ In this approximation, the above formula is simplified as follows:

$$
\langle O[z]\rangle=\langle O[z]\rangle_{\mathcal{J}_{\text {vac }}}
$$

We then summarize a few geometric properties of Lefschetz thimbles. First we recall that for a given critical point $z_{\sigma} \in \Sigma$, the associated thimble $\mathcal{J}_{\sigma}$ is the union of all downward flows which trace back to $z_{\sigma}$ at $t=-\infty$. In the vicinity of the cirtical point $z_{\sigma}$, the flow equation eq. (2.2) can be linearized $a^{9}$

$$
\frac{d}{d t}\left(z_{i}(t)-z_{\sigma i}\right)=\bar{K}_{i j}\left(\bar{z}_{j}(t)-\bar{z}_{\sigma j}\right),\left.\quad K_{i j} \equiv \partial_{i} \partial_{j} S[z]\right|_{z=z_{\sigma}} .
$$

The complex symmetric matrix $K_{i j}$, according to the Takagi factorization theorem[39], can be cast into a positive diagonal matrix as $v_{i}^{\alpha} K_{i j} v_{j}^{\beta}=\kappa^{\alpha} \delta^{\alpha \beta}$, where $\kappa^{\alpha} \geq 0(\alpha=1, \cdots, n)$

\footnotetext{
${ }^{8}$ While $\langle O[z]\rangle_{\mathcal{J}_{\sigma}}$ may be evaluated through Monte Carlo simulations as discussed in [11, 36] and will be discussed in the following sections, it is not straightforward to compute $\left\{Z_{\sigma}\right\}(\sigma \in \Sigma)$ in general. At one-loop, i.e. in the saddle point approximation, $Z_{\sigma}=1 / \sqrt{\operatorname{det} K}$ where $K$ is defined in eq. (2.9) below.

${ }^{9}$ In the following, we will use the abbreviation $\partial / \partial z_{i}=\partial_{i}, \bar{\partial} / \partial \bar{z}_{i}=\bar{\partial}_{i}$.
} 
and $v_{i}^{\alpha}(\alpha=1, \cdots, n)$ are orthonormal complex vectors. And the solution to the linearized flow equation is obtained as

$$
z_{i}(t)-z_{\sigma i}=v_{i}^{\alpha} \exp \left(\kappa^{\alpha}\left(t-t_{0}\right)\right) \xi_{0}^{\alpha}, \quad \xi_{0}^{\alpha} \in \mathbb{R}(\alpha=1, \cdots, n) .
$$

Indeed, the set of the orthonormal vectors $\left\{v^{\alpha}\right\}(\alpha=1, \cdots, n)$ spans the tangent space of the Lefschetz thimble $\mathcal{J}_{\sigma}$ at the critical point $z_{\sigma}, T_{z_{\sigma}}$ : close to the critical point, the thimble is parametrized by $n$ real parameters $\xi^{\alpha} \in \mathbb{R}(\alpha=1, \cdots, n)$ as $z_{i}-z_{\sigma i} \simeq v_{i}^{\alpha} \xi^{\alpha}$, and the action reads $S[z]-S\left[z_{\sigma}\right] \simeq\left(z_{i}-z_{\sigma i}\right) K_{i j}\left(z_{j}-z_{\sigma j}\right) / 2=\kappa^{\alpha} \xi^{\alpha} \xi^{\alpha} / 2 \in \mathbb{R}$.

At a generic point $z$ on the thimble $\mathcal{J}_{\sigma}$, one can also define a tangent space $T_{z}$ and a basis of tangent vectors $\left\{V_{z}^{\alpha}\right\}(\alpha=1, \cdots, n)$. Because any two tangent vectors $V_{z}$ and $V_{z}^{\prime}$ should commute with each other, $\left\{V_{z} \partial+\bar{V}_{z} \bar{\partial}\right\} V_{z}^{\prime}-\left\{V_{z}^{\prime} \partial+\bar{V}_{z}^{\prime} \bar{\partial}\right\} V_{z}=0$, and the direction vector of the gradient flow, $g \equiv \bar{\partial} \bar{S}[\bar{z}]$, itself should be a tangent vector, it follows that $\left\{V_{z}^{\alpha}\right\}$ satisfy the following flow equations, ${ }^{10} 11$

$$
\frac{d}{d t} V_{z i}^{\alpha}(t)=\bar{\partial}_{i} \bar{\partial}_{j} \bar{S}[\bar{z}] \bar{V}_{z j}^{\alpha}(t) \quad(\alpha=1, \cdots, n) .
$$

Indeed, $g \equiv \bar{\partial} \bar{S}[\bar{z}]$ itself satisfies this flow equation and it is expanded in terms of $\left\{V_{z}^{\alpha}\right\}$ as $g=\bar{\partial} \bar{S}[\bar{z}]=V_{z}^{\alpha} g^{\alpha}$ with $n$ real constants $g^{\alpha} \in \mathbb{R}(\alpha=1, \cdots, n)$. It also follows that $\left\{V_{z}^{\alpha}\right\}$ satisfy a reality condition, ${ }^{12}$

$$
\bar{V}_{z i}^{\alpha} V_{z i}^{\beta}-\bar{V}_{z i}^{\beta} V_{z i}^{\alpha}=0 \quad(\alpha, \beta=1, \cdots, n) .
$$

The basis of tangent vectors $\left\{V_{z}^{\alpha}\right\}$, which satisfy the flow equations eq. (2.11), is not orthonormal in general. One can make it orthonormal by Gram-Schmidt orthonormalization, or Iwasawa decomposition. In fact, $\left\{V_{z}^{\alpha}\right\}$ can be expressed in the following form,

$$
V_{z}^{\alpha}=U_{z}^{\beta} E^{\beta \alpha}
$$

${ }^{10}$ The commutation relation of two vectors $V_{z}^{\alpha}$ and $V_{z}^{\beta}$, if one of the vectors is set to the direction vector of the Lefschetz flow $g \equiv \bar{\partial} \bar{S}[\bar{z}]$, reads $\{g \partial+\bar{g} \bar{\partial}\} V_{z}^{\alpha}-\left\{V_{z}^{\alpha} \partial+\bar{V}_{z}^{\alpha} \bar{\partial}\right\} g=0$. This immediately implies that

$$
\frac{d}{d t} V_{z i}^{\alpha}(t)=\left\{V_{z}^{\alpha} \partial+\bar{V}_{z}^{\alpha} \bar{\partial}\right\} g_{i}=\bar{\partial}_{i} \bar{\partial}_{j} \bar{S}[\bar{z}] \bar{V}_{z j}^{\alpha}(t) .
$$

${ }^{11}$ In the vicinity of the critical point $z_{\sigma}$, the flow equation for the tangent vectors eq. (2.11) is linearized as $d V_{i}^{\alpha}(t) / d t=\bar{K}_{i j} \bar{V}_{j}^{\alpha}(t)$. And the solution to the equation is obtained as

$$
V_{i}^{\alpha}(t)=v_{i}^{\beta} \exp \left(\kappa^{\beta}\left(t-t_{0}\right)\right) C_{0}^{\beta \alpha}, \quad C_{0}^{\beta \alpha} \in \mathbb{R}(\alpha, \beta=1, \cdots, n) .
$$

Without loss of generality, one can set $\mathrm{e}^{-\kappa^{\beta} t_{0}} C_{0}^{\beta \alpha}=\delta^{\beta \alpha}$.

12 To show the reality condition, one should note

$$
\frac{d}{d t} \operatorname{Im}\left\{\bar{V}_{z}^{\alpha}(t) V_{z}^{\beta}(t)\right\}=\operatorname{Im}\left\{V_{z}^{\alpha} \partial^{2} S[z] V_{z}^{\beta}(t)+\bar{V}_{z}^{\alpha} \bar{\partial}^{2} \bar{S}[\bar{z}] \bar{V}_{z}^{\beta}(t)\right\}=0,
$$

and

$$
\operatorname{Im}\left\{\bar{V}_{z}^{\alpha}(t) V_{z}^{\beta}(t)\right\}=\operatorname{Im}\left\{\bar{v}^{\alpha} v^{\beta}\right\} \exp \left(\kappa^{\alpha} t\right) \exp \left(\kappa^{\beta} t\right)=0 \quad(t \ll 0) .
$$


where $\left\{U_{z}^{\alpha}\right\}$ is a orthonormal basis and $E$ is a real upper triangle matrix. ${ }^{13}$ In the vicinity of $z$, therefore, the thimble can be parametrized by real orthogonal coordinates $\left\{\delta \xi^{\alpha}\right\}(\alpha=$ $1, \cdots, n)$ such that $\delta z=U_{z}^{\alpha} \delta \xi^{\alpha},|\delta z|^{2}=\delta \xi^{2}$, and $\left.d^{n} z\right|_{\mathcal{J}_{\sigma}}=d^{n} \delta \xi \operatorname{det} U_{z}$. Thus the measure on the thimbles, $\mathcal{D}[z]=\left.d^{n} z\right|_{\mathcal{J}_{\sigma}}$, gives rise to an extra complex phase defined by

$$
\mathrm{e}^{i \phi_{z}}=\operatorname{det} U_{z}=\frac{\operatorname{det} V_{z}}{\left|\operatorname{det} V_{z}\right|}
$$

Given the tangent space $T_{z}$ and the basis of tangent vectors $\left\{V_{z}^{\alpha}\right\}(\alpha=1, \cdots, n)$, directions normal to the thimble at $z \in \mathcal{J}_{\sigma}$ are determined by the set of normal vectors $\left\{i U_{z}^{\alpha}\right\}$ or $\left\{i V_{z}^{\alpha}\right\}(\alpha=1, \cdots, n)$. This is because the reality condition eq. (2.12) implies that

$$
\operatorname{Re}\left\{(-i) \bar{V}_{z i}^{\alpha} V_{z i}^{\beta}\right\}=0 \quad(\alpha, \beta=1, \cdots, n),
$$

and $\left\{i V_{z}^{\alpha}\right\}$ are orthogonal to $\left\{V_{z}^{\beta}\right\}$ with respect to the inner product in $\mathbb{R}^{2 n}$.

Finally, any point $z$ on the thimble $\mathcal{J}_{\sigma}$ is identified uniquely by the direction of the flow on which $z$ lies and the time of the flow to get to $z$, both defined referring to a certain asymptotic region close to the critical point. In fact, the asymptotic solutions to the flow equations eqs. (2.2) and (2.11) for $t \ll 0$ can be expressed without loss of generality by

$$
\begin{aligned}
& z(t) \simeq z_{\sigma}+v^{\alpha} \exp \left(\kappa^{\alpha} t\right) e^{\alpha} ; \quad e^{\alpha} e^{\alpha}=n, \\
& V_{z}^{\alpha}(t) \simeq v^{\alpha} \exp \left(\kappa^{\alpha} t\right),
\end{aligned}
$$

and one can define the direction of the flow by $e^{\alpha}\left(\alpha=1, \cdots, n ;\|e\|^{2}=n\right)$ and the time of the flow by $t^{\prime}=t-t_{0}$ with a certain reference time $t_{0} \ll 0 .{ }^{14}$ One can then define a map $z\left[e, t^{\prime}\right]:\left(e^{\alpha}, t^{\prime}\right) \rightarrow z \in \mathcal{J}_{\sigma}$ by

$$
z\left[e, t^{\prime}\right]=\left.z(t)\right|_{t=t^{\prime}+t_{0}},
$$

provided the asymptotic form of the flow $z(t)$ is given by eq. (2.16). ${ }^{15}$ Moreover, under infinitesimal variations of the parameters $\left(e^{\alpha}, t^{\prime}\right)$, the variation of $z\left[e, t^{\prime}\right]$ is given by the following formula,

$$
\delta z\left[e, t^{\prime}\right]=V_{z}^{\alpha}\left[e, t^{\prime}\right]\left(\delta e^{\alpha}+\kappa^{\alpha} e^{\alpha} \delta t^{\prime}\right) .
$$

This is because an infinitesimal variation of the flow $\delta z(t)$ itself satisfies the flow equation for a tangent vector,

$$
\delta \dot{z}_{i}(t)=\bar{\partial}_{i} \bar{\partial}_{j} \bar{S}[\bar{z}] \delta z_{j}(t)
$$

\footnotetext{
${ }^{13}$ By the Iwasawa decomposition, $V_{z}$ can be expressed in the form $V_{z}=U_{z} D N$, where $U_{z}$ is unitary, $D$ is positive diagonal, and $N$ is upper triangle with the unit diagonal elements. But, from the property $\bar{V}_{z i}^{\alpha} V_{z i}^{\beta}=\bar{V}_{z i}^{\beta} V_{z i}^{\alpha}$, one can show further that $N$ is real. Therefore, there exists a real upper triangle matrix $E=D N$, and the tangent vectors $\left\{V_{z}^{\alpha}\right\}$ are related to the orthonormal tangent vectors $\left\{U_{z}^{\alpha}\right\}$ by $V_{z}^{\alpha}=$ $U_{z}^{\beta} E^{\beta \alpha}$.

${ }^{14} t_{0}$ should be chosen so that $\|\epsilon\|^{2} \ll n$ where $\epsilon^{\alpha} \equiv \exp \left(\kappa^{\alpha} t_{0}\right) e^{\alpha}$ and the linear approximation of the flow equation is valid.

${ }^{15}$ In [37], a similar map between a thimble and its asymptotic "Gaussian" region has been introduced.
} 
and it should be expanded in terms of $\left\{V_{z}^{\alpha}\right\}$ as $\delta z(t)=V_{z}^{\alpha}(t) \delta c^{\alpha}$ with constants $\delta c^{\alpha} \in$ $\mathbb{R}(\alpha=1, \cdots, n)$. These constants $\left\{\delta c^{\alpha}\right\}$ may be determined from the asymptotic form of $\delta z(t)$ for $t \ll 0$,

$$
\begin{aligned}
\delta z(t) & =v^{\alpha} \exp \left(\kappa^{\alpha} t\right)\left(\delta e^{\alpha}+e^{\alpha} \kappa^{\alpha} \delta t\right) & \\
& =V_{z}^{\alpha}(t)\left(\delta e^{\alpha}+\kappa^{\alpha} e^{\alpha} \delta t\right) & (t \ll 0),
\end{aligned}
$$

and one obtains $\delta c^{\alpha}=\delta e^{\alpha}+\kappa^{\alpha} e^{\alpha} \delta t^{16}$

\section{An algorithm of hybrid Monte Carlo on Lefschetz thimbles}

Next we describe a hybrid Monte Carlo algorithm which is applicable to the lattice models defined on Lefschetz thimbles. Because of saddle-point structures of Lefschetz thimbles, it is not easy to keep track of a thimble in stochastic processes like Langevin and hybrid Monte Carlo updates. It is then necessary to be able to locate a field configuration on the thimble, $z \in \mathcal{J}_{\sigma}$, precisely in $\mathbb{C}^{n}$ and to constrain the field configuration onto the thimble in every stochastic step. We therefore parametrize any point on the thimble $z \in \mathcal{J}_{\sigma}$ uniquely by the direction of the flow $e^{\alpha}\left(\alpha=1, \cdots, n ;\|e\|^{2}=n\right)$ and the time of the flow $t^{\prime}=t-t_{0}$ with a fixed reference time $t_{0}(\ll 0)$, as discussed in the previous section. Moreover, regarding the molecular dynamics in hybrid Monte Carlo, we consider a constrained dynamical system including the forces normal to the thimble, i.e. along the normal vectors $\left\{i V_{z}^{\alpha}\right\}(\alpha=1, \cdots, n)$, with Lagrange multipliers. To integrate the equations of motion of the constrained system, we employ a second order constraint-preserving symmetric integrator[38].

\subsection{To generate a thimble by solving the flow equations downward}

For given parameters $\left(e^{\alpha}, t^{\prime}\right)$ and $t_{0}(\ll 0)$, we generate the point $z\left[e, t^{\prime}\right] \in \mathcal{J}_{\sigma}$ by solving the flow equations eqs. (2.2) and (2.11) downward with the initial conditions,

$$
\begin{aligned}
z_{i}\left(t_{0}\right) & =z_{\sigma i}+v_{i}^{\alpha} \exp \left(\kappa^{\alpha} t_{0}\right) e^{\alpha} \\
V_{z i}^{\alpha}\left(t_{0}\right) & =v_{i}^{\alpha} \exp \left(\kappa^{\alpha} t_{0}\right) .
\end{aligned}
$$

We employ the fourth-order Runge-Kutta method with the number of iterations $n_{\text {lefs }}$ and the size of increment $h \equiv t^{\prime} / n_{\text {lefs. }}{ }^{17}$

To verify the solutions, one may check if the following relation is satisfied:

$$
\bar{\partial}_{i} \bar{S}\left[\bar{z}\left[e, t^{\prime}\right]\right]-V_{z i}^{\alpha}\left[e, t^{\prime}\right] \kappa^{\alpha} e^{\alpha}=0 .
$$

In what precision this relation holds would depend on several conditions and parameters. First of all, it depends on the sizes of $\left\|z\left(t_{0}\right)-z_{\sigma}\right\|$ and $\operatorname{Re}\left(S\left[z\left(t_{0}\right)\right]-S\left[z_{\sigma}\right]\right)$, which indicate how close to the critical point $z_{\sigma}$ the reference point $z\left(t_{0}\right)$ is. It depends also on the

\footnotetext{
${ }^{16}$ In a similar reasoning, one obtains $g^{\alpha}=\kappa^{\alpha} e^{\alpha}$, i.e. $g=\bar{\partial} \bar{S}[\bar{z}]=V_{z}^{\alpha} \kappa^{\alpha} e^{\alpha}$.

17 The computation of the tangent vectors $\left\{V_{z}^{\alpha}\right\}(\alpha=1, \cdots, n)$ is numerically very demanding. We have used GPUs in executing this computation.
} 
parameters of the Runge-Kutta method, $n_{\text {lefs }}$ and $h \equiv t^{\prime} / n_{\text {lefs }}$, and the size of the system, $n$.

Once the matrix $V_{z}=\left(V_{z i}^{\alpha}\right)$ is obtained, its inverse $V_{z}^{-1}=\left(\left\{V_{z}^{-1}\right\}_{i}^{\alpha}\right)$ such that $\sum_{\beta} V_{z i}^{\beta}\left\{V_{z}^{-1}\right\}_{j}^{\beta}=\delta_{i j}$ and its determinant $\operatorname{det} V_{z}$ are computed through LU decomposition.

\subsection{Constrained molecular dynamics}

To formulate the molecular dynamics on the thimble $\mathcal{J}_{\sigma}$, we introduce a dynamical system defined by the equations of motion, ${ }^{18}$

$$
\begin{aligned}
\dot{z}_{i} & =w_{i}, \\
\dot{w}_{i} & =-\bar{\partial}_{i} \bar{S}[\bar{z}]-i V_{z i}^{\alpha} \lambda^{\alpha},
\end{aligned}
$$

and the constraints,

$$
z_{i}=z_{i}\left[e, t^{\prime}\right]
$$

where $w_{i}$ are the momenta conjugate to $z_{i}$ and $\lambda^{\alpha} \in \mathbb{R}(\alpha=1, \cdots, n)$ are the Lagrange multipliers. ${ }^{19}$ It follows from the equations of motion eqs. (3.4), (3.5) and the constraint eq. (3.6) that

$$
w_{i}=V_{z i}^{\alpha}\left[e, t^{\prime}\right] w^{\alpha}, \quad w^{\alpha} \in \mathbb{R} \quad \text { or } \quad \operatorname{Im}\left[\left\{V_{z}^{-1}\right\}_{j}^{\alpha} w_{j}\right]=0 .
$$

In this system, a conserved Hamiltonian is given by

$$
H=\frac{1}{2} \bar{w}_{i} w_{i}+\frac{1}{2}\{S[z]+\bar{S}[\bar{z}]\}
$$

It follows indeed that

$$
\begin{aligned}
\dot{H} & =\frac{1}{2}\left\{\dot{\bar{w}}_{i} w_{i}+\bar{w}_{i} \dot{w}_{i}\right\}+\frac{1}{2}\left\{\partial_{i} S[z] \dot{z}_{i}+\bar{\partial}_{i} \bar{S}[\bar{z}] \dot{\bar{z}}_{i}\right\} \\
& =\frac{1}{2}\left\{\left(+i \bar{V}_{z i}^{\alpha} \lambda^{\alpha}\right) w_{i}+\bar{w}_{i}\left(-i V_{z i}^{\alpha} \lambda^{\alpha}\right)\right\} \\
& =\frac{i}{2} \lambda^{\alpha} w^{\beta}\left\{\bar{V}_{z i}^{\alpha} V_{z i}^{\beta}-\bar{V}_{z i}^{\beta} V_{z i}^{\alpha}\right\}=0 .
\end{aligned}
$$

To integrate the equations of motion with the Lagrange multipliers eqs. (3.4) and (3.5), we employ the second order constraint-preserving symmetric integrator[38]: it is assumed first that $z^{n}$ and $w^{n}$ satisfy the constraints

$$
\begin{aligned}
z^{n} & =z\left[e^{(n)}, t^{\prime(n)}\right], \\
w^{n} & =V_{z}^{\alpha}\left[e^{(n)}, t^{\prime(n)}\right] w^{\alpha(n)}, \quad w^{\alpha(n)} \in \mathbb{R},
\end{aligned}
$$

\footnotetext{
${ }^{18}$ We use the abbreviation, $\frac{d}{d \tau} y(\tau)=\dot{y}$, where $\tau$ denotes the time coordinate of the dynamical system.

19 The molecular dynamics on Lefschetz thimbles may be formulated by a Hamilton system on Riemann manifolds[40]. For example, one may introduce auxiliary dynamical variables $x^{\alpha} \equiv \exp \left(\kappa^{\alpha}\left(t^{\prime}+t_{0}\right)\right) e^{\alpha}$ and the metric $G^{\alpha \beta}[x] \equiv V_{z i}^{\alpha}\left[e, t^{\prime}\right] \bar{V}_{z i}^{\beta}\left[e, t^{\prime}\right] \exp \left(-\kappa^{\alpha}\left(t^{\prime}+t_{0}\right)\right) \exp \left(-\kappa^{\beta}\left(t^{\prime}+t_{0}\right)\right)$ so that $\|\delta z\|^{2}=G^{\alpha \beta}[x] \delta x^{\alpha} \delta x^{\beta}$. One may then consider the Hamilton system with a non-separable Hamiltonian,

$$
H=\frac{1}{2}\left\{G^{-1}\right\}^{\alpha \beta}[x] p^{\alpha} p^{\beta}+\frac{1}{2}\{S+\bar{S}\}[x]+\frac{1}{2} \operatorname{Tr} \operatorname{Ln}(G[x]) .
$$

The equations of motion of this system may be solved by an implicit second order symplectic integrator. 
and $z^{n+1}$ and $w^{n+1}$ are then determined for a given step size $\Delta \tau$ by

$$
\begin{aligned}
w^{n+1 / 2} & =w^{n} \quad-\frac{1}{2} \Delta \tau \bar{\partial} \bar{S}\left[\bar{z}^{n}\right] \quad-\frac{1}{2} \Delta \tau i V_{z}^{\alpha}\left[e^{(n)}, t^{\prime(n)}\right] \lambda_{[r]}^{\alpha}, \\
z^{n+1} & =z^{n} \quad+\Delta \tau w^{n+1 / 2}, \\
w^{n+1} & =w^{n+1 / 2}-\frac{1}{2} \Delta \tau \bar{\partial} \bar{S}\left[\bar{z}^{n+1}\right]-\frac{1}{2} \Delta \tau i V_{z}^{\alpha}\left[e^{(n+1)}, t^{\prime(n+1)}\right] \lambda_{[v]}^{\alpha},
\end{aligned}
$$

where $\lambda_{[r]}^{\alpha}$ and $\lambda_{[v]}^{\alpha}$ are fixed by imposing the constraints,

$$
\begin{aligned}
& z^{n+1}=z\left[e^{(n+1)}, t^{\prime(n+1)}\right], \\
& w^{n+1}=V_{z}^{\alpha}\left[e^{(n+1)}, t^{\prime(n+1)}\right] w^{\alpha(n+1)}, \quad w^{\alpha(n+1)} \in \mathbb{R},
\end{aligned}
$$

respectively. The first constraint eq. (3.16) reads

$$
\begin{aligned}
z\left[e^{(n+1)}, t^{\prime(n+1)}\right]-z\left[e^{(n)}, t^{\prime(n)}\right]=\Delta \tau w^{n} & -\frac{1}{2} \Delta \tau^{2} \bar{\partial} \bar{S}\left[\bar{z}^{n}\right] \\
& -\frac{1}{2} \Delta \tau^{2} i V_{z}^{\alpha}\left[e^{(n)}, t^{\prime(n)}\right] \lambda_{[r]}^{\alpha} .
\end{aligned}
$$

This is solved by a fixed-point iteration $\operatorname{method}^{20}$ : to find $\left(e^{\alpha(n+1)}, t^{\prime(n+1)}\right)$ and $\lambda_{[r]}^{\alpha}$, we generate the sequences $\left(e_{(k)}^{\alpha}, t_{(k)}^{\prime}\right)(k=0,1, \cdots)$ with $\left(e_{(0)}^{\alpha}, t_{(0)}^{\prime}\right)=\left(e^{\alpha(n)}, t^{\prime(n)}\right)$ and $\lambda_{[r]}^{\alpha}(k)$ $0,1, \cdots)$ so that the increments,

$$
\begin{aligned}
& \Delta e_{(k)}^{\alpha}=e_{(k+1)}^{\alpha}-e_{(k)}^{\alpha}, \quad \sum_{\alpha=1}^{n} \Delta e_{(k)}^{\alpha} e^{\alpha(n)}=0, \\
& \Delta t_{(k)}^{\prime}=t_{(k+1)}^{\prime}-t_{(k)}^{\prime},
\end{aligned}
$$

are infinitesimal and $\left(\Delta e^{\alpha}{ }_{(k)}, \Delta t_{(k)}^{\prime}\right)$ and $\lambda_{[r]_{(k)}}^{\alpha}$ are determined by

$$
\begin{aligned}
& \Delta e_{(k)}^{\alpha}+e^{\alpha(n)} \kappa^{\alpha} \Delta t_{(k)}^{\prime}=\operatorname{Re}\left[\left\{V_{z}^{-1}\left[e^{(n)}, t^{\prime(n)}\right]\right\}_{i}^{\alpha} \times\right. \\
&\left.\left(z_{i}\left[e^{(n)}, t^{\prime(n)}\right]+\Delta \tau w_{i}^{n}-\frac{1}{2} \Delta \tau^{2} \bar{\partial}_{i} \bar{S}\left[\bar{z}^{n}\right]-z_{i}\left[e_{(k)}, t_{(k)}^{\prime}\right]\right)\right] \\
&\left.\frac{1}{2} \Delta \tau^{2} \lambda_{[r]}^{\alpha}\right]_{(k)}=\operatorname{Im}\left[\left\{V_{z}^{-1}\left[e^{(n)}, t^{\prime(n)}\right]\right\}_{i}^{\alpha}\left(z_{i}\left[e^{(n)}, t^{\prime(n)}\right]-z_{i}\left[e_{(k)}, t_{(k)}^{\prime}\right]\right)\right]
\end{aligned}
$$

until a stopping condition,

$$
\left\|V_{z}^{\alpha}\left[e^{(n)}, t^{\prime(n)}\right]\left(\Delta e_{(k)}^{\alpha}+e^{\alpha(n)} \kappa^{\alpha} \Delta t_{(k)}^{\prime}\right)\right\|^{2} \leq n \epsilon^{\prime 2},
$$

is satisfied for a sufficiently small $\epsilon^{\prime}$ to achieve a given precision. ${ }^{21}$ (See fig. 1.) Once $\left(e^{\alpha(n+1)}, t^{\prime(n+1)}\right)$ and $z\left[e^{(n+1)}, t^{\prime(n+1)}\right]$ are obtained, we compute the set of tangent vectors $\left\{V_{z}^{\alpha}\left[e^{(n+1)}, t^{\prime(n+1)}\right]\right\}$ and the inverse matrix $V_{z}^{-1}\left[e^{(n+1)}, t^{\prime(n+1)}\right]$. The second constraint in eq. (3.17) is then solved by

$$
\frac{1}{2} \Delta \tau \lambda_{[v]}^{\alpha}=\operatorname{Im}\left[\left\{V_{z}^{-1}\left[e^{(n+1)}, t^{(n+1)}\right]\right\}_{i}^{\alpha}\left(w_{i}^{n+1 / 2}-\frac{1}{2} \Delta \tau \bar{\partial}_{i} \bar{S}\left[\bar{z}^{n+1}\right]\right)\right] .
$$

\footnotetext{
${ }^{20}$ This method to find $\left(e^{\alpha(n+1)}, t^{\prime(n+1)}\right)$ and $\lambda_{[r]}^{\alpha}$ in eq. (3.18) can also be used in Langevin-type updates.

${ }^{21}$ The squared norm of $e_{(k+1)}^{\alpha}$ has the second order correction, $\left\|e_{(k+1)}^{\alpha}\right\|^{2}=\left\|e_{(k)}+\Delta e_{(k)}\right\|^{2}=n+\left(\Delta e_{(k)}\right)^{2}$, and it is renormalized as $e_{(k+1)}^{\alpha} \rightarrow e_{(k+1)}^{\alpha} / \sqrt{1+\left(\Delta e_{(k)}\right)^{2} / n}$.
} 


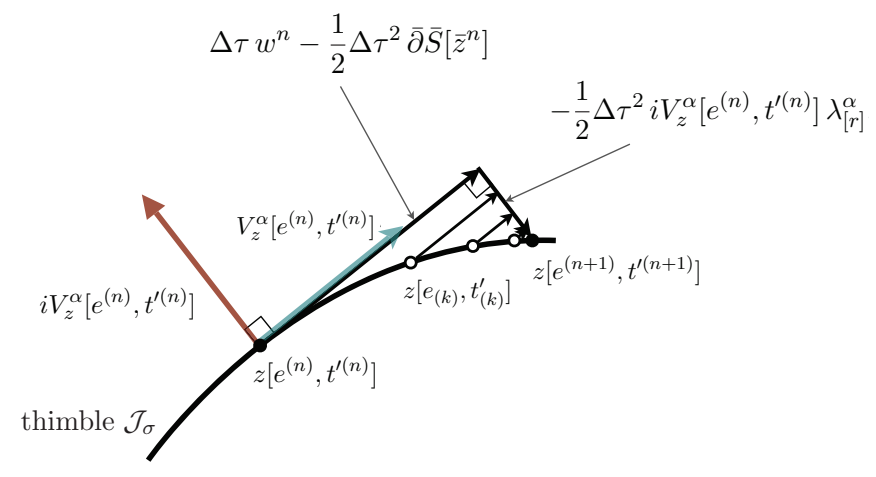

Figure 1. A fixed-point method to solve the constraint eq. (3.16).

\subsection{Hybrid Monte Carlo updates}

A hybrid Monte Carlo update then consists of the following steps for a given trajectory length $\tau_{\text {traj }}$ and a number of steps $n_{\text {step }}$ :

1. Set the initial field configuration $z_{i}$ :

$$
\left\{e^{\alpha(0)}, t^{\prime(0)}\right\}=\left\{e^{\alpha}, t^{\prime}\right\}, \quad z^{0}=z\left[e, t^{\prime}\right]
$$

2. Refresh the momenta $w_{i}$ by generating $n$ pairs of unit gaussian random numbers $\left(\xi_{i}, \eta_{i}\right)$, setting tentatively $w_{i}=\xi_{i}+i \eta_{i}$, and chopping the non-tangential parts:

$$
w^{0}=V_{z}^{\alpha} \operatorname{Re}\left[\left\{V_{z}^{-1}\right\}_{j}^{\alpha}\left(\xi_{j}+i \eta_{j}\right)\right]=U_{z}^{\alpha} \operatorname{Re}\left[\left\{U_{z}^{-1}\right\}_{j}^{\alpha}\left(\xi_{j}+i \eta_{j}\right)\right] .
$$

3. Repeat $n_{\text {step }}$ times of the second order symmetric integration eqs. (3.13)-(3.17) with the step size $\Delta \tau=\tau_{\text {traj }} / n_{\text {step }}$.

4. Accept or reject by $\Delta H=H\left[w^{n_{\text {step }}}, z^{n_{\text {step }}}\right]-H\left[w^{0}, z^{0}\right]$.

As for the initialization procedure, one may generate unit gaussian random numbers $\eta^{\alpha}(\alpha=1, \cdots, n)$, set

$$
e^{\alpha}=\eta^{\alpha} \sqrt{\frac{n}{\sum_{b=1}^{n} \eta^{b} \eta^{b}}}, \quad t^{\prime}=-t_{0},
$$

and then prepare $z\left[e, t^{\prime}\right],\left\{V_{z}^{\alpha}\left[e, t^{\prime}\right]\right\}$, and the inverse matrix $V_{z}^{-1}\left[e, t^{\prime}\right]$.

\subsection{To measure observables by reweighting the residual sign factors}

In the hybrid Monte Carlo method described above, the contribution of the residual phase factor, $\mathrm{e}^{i \phi_{z}}=\operatorname{det} V_{z} /\left|\operatorname{det} V_{z}\right|$, is neglected. To obtain the expectation value of an observable on the given thimble $\mathcal{J}_{\sigma}$, we need to evaluate the average of the observable with the residual 
phase factor reweighed. Let us denote the simple statistical average of an operator $o[z]$ on the thimble $\mathcal{J}_{\sigma}$ by $\langle o[z]\rangle_{\mathcal{J}_{\sigma}}^{\prime}$ :

$$
\langle o[z]\rangle_{\mathcal{J}_{\sigma}}^{\prime}=\frac{1}{N_{\text {conf }}} \sum_{k=1}^{N_{\text {conf }}} o\left[z^{(k)}\right],
$$

where $N_{\text {conf }}$ is the number of field configurations obtained by the hybrid Monte Carlo updates. The expectation value of a given observable $O[z]$ on the thimble $\mathcal{J}_{\sigma}$ should then be evaluated by the following formula,

$$
\langle O[z]\rangle_{\mathcal{J}_{\sigma}}=\frac{\left\langle\mathrm{e}^{i \phi_{z}} O[z]\right\rangle_{\mathcal{J}_{\sigma}}^{\prime}}{\left\langle\mathrm{e}^{i \phi_{z}}\right\rangle_{\mathcal{J}_{\sigma}}^{\prime}} .
$$

For this formula eq. (3.29) to work, it is crucial that the averages of the residual sign factors, $\left\{\left\langle\mathrm{e}^{i \phi_{z}}\right\rangle_{\mathcal{J}_{\sigma}}^{\prime}\right\}(\sigma \in \Sigma)$, are not vanishingly small, in particular, for the thimble associated with the classical vacuum, $\mathcal{J}_{\text {vac }}$. This is the possible sign problem in our hybrid Monte Carlo method, which should be studied carefully and systematically.

\section{HMC simulations of the complexified $\lambda \phi^{4}$ model at finite density}

Now we test the hybrid Monte Carlo algorithm described in the previous section by applying it to the complex $\lambda \phi^{4}$ model with chemical potential $\mu[17,32,36]$. The action of the model is defined in the lattice unit by

$$
\begin{aligned}
S=\sum_{x \in \mathbb{L}^{4}} & \left\{\left(\varphi^{\dagger}(x+\hat{0}) \mathrm{e}^{+\mu}-\varphi^{\dagger}(x)\right)\left(\mathrm{e}^{-\mu} \varphi(x+\hat{0})-\varphi(x)\right)\right. \\
& \left.+\sum_{k=1}^{3}|\varphi(x+\hat{k})-\varphi(x)|^{2}+\frac{\kappa}{2} \varphi^{\dagger}(x) \varphi(x)+\frac{\lambda}{4}\left(\varphi^{\dagger}(x) \varphi(x)\right)^{2}\right\} \\
=\sum_{x \in \mathbb{L}^{4}} & \left\{-\phi_{a}(x) \phi_{b}(x+\hat{0})\left[\delta_{a b} \cosh (\mu)-i \epsilon_{a b} \sinh (\mu)\right]\right. \\
& \left.-\sum_{k=1}^{3} \phi_{a}(x) \phi_{a}(x+\hat{k})+\frac{(8+\kappa)}{2} \phi_{a}(x) \phi_{a}(x)+\frac{\lambda}{4}\left(\phi_{a}(x) \phi_{a}(x)\right)^{2}\right\},
\end{aligned}
$$

where $\varphi(x)=\left(\phi_{1}(x)+i \phi_{2}(x)\right) / \sqrt{2}$ and the real field variables $\phi_{a}(x) \in \mathbb{R}(a=1,2)$ are used in the second expression. We assume that the lattice $\mathbb{L}^{4}$ is finite with a linear extent $L$ and a volume $V=L^{4}$, and the field variables satisfy the periodic boundary conditions. In complexification, the field variables are complexified as $\phi_{a}(x) \rightarrow z_{a}(x) \in \mathbb{C}(a=1,2)$ and rescaled for later convenience as $z_{a}(x) \rightarrow \sqrt{K_{0}} z_{a}(x)$ so that $K_{0}(8+\kappa)=1$ and $K_{0}^{2} \lambda=\lambda_{0}$. The complexified action then reads

$$
\begin{aligned}
S[z]=\sum_{x \in \mathbb{L}^{4}}\{ & +\frac{1}{2} z_{a}(x) z_{a}(x)+\frac{\lambda_{0}}{4}\left(z_{a}(x) z_{a}(x)\right)^{2}-K_{0} \sum_{k=1}^{3} z_{a}(x) z_{a}(x+\hat{k}) \\
& \left.-K_{0} z_{a}(x) z_{b}(x+\hat{0})\left[\delta_{a b} \cosh (\mu)-i \epsilon_{a b} \sinh (\mu)\right]\right\} .
\end{aligned}
$$


Among possible critical points in this model, those with constant fields $z_{a}(x)=z_{a}$ are relatively easy to find. Such critical points are determined by the following stationary condition,

$$
\left.\frac{\partial S[z]}{\partial z_{a}(x)}\right|_{z_{a}(x)=z_{a}}=\left(1-6 K_{0}-2 K_{0} \cosh (\mu)\right) z_{a}+\lambda_{0}\left(z_{1}^{2}+z_{2}^{2}\right) z_{a}=0 \quad(a=1,2) .
$$

There is a classical critical value in $\mu$, for fixed $K_{0}(<1 / 8)$ and $\lambda_{0}(>0)$, given by

$$
\tilde{\mu}_{c}=\ln \left[\left(\frac{1-6 K_{0}}{2 K_{0}}\right)+\sqrt{\left(\frac{1-6 K_{0}}{2 K_{0}}\right)^{2}-1}\right]
$$

and the solutions to the stationary condition are obtained as follows:

1. For $\mu \leq \tilde{\mu}_{c}$,

(a) $z_{1}=z_{2}=0 ; S[z]=0$,

(b) $z_{1}=i \phi_{0} \cos \theta, z_{2}=i \phi_{0} \sin \theta ; S[z]=-L^{4} \frac{\lambda_{0}}{4} \phi_{0}^{4}$,

where $\phi_{0}=\sqrt{\frac{+\left(1-6 K_{0}-2 K_{0} \cosh (\mu)\right)}{\lambda_{0}}}$.

2. For $\mu>\tilde{\mu}_{c}$,

(a) $z_{1}=z_{2}=0 ; \quad S[z]=0$,

(b) $z_{1}=\phi_{0} \cos \theta, z_{2}=\phi_{0} \sin \theta ; S[z]=-L^{4} \frac{\lambda_{0}}{4} \phi_{0}^{4}$,

where $\phi_{0}=\sqrt{\frac{-\left(1-6 K_{0}-2 K_{0} \cosh (\mu)\right)}{\lambda_{0}}}$.

The solutions 1-(a), 2-(a), and 2-(b) are real. They are in fact the classical solutions in the original model, and the solutions 1-(a) and 2-(b) are the classical vacua for $\mu<\tilde{\mu}_{c}$ and $\mu>\tilde{\mu}_{c}$, respectively. The solution 1-(b) are pure imaginary, and the thimbles associated with this critical point do not contribute to the path-integration, because $-\operatorname{Re} S\left[z_{\sigma}\right]>$ $\max \{-\operatorname{Re} S[x]\}\left(=0\right.$ for $\left.\mu<\tilde{\mu}_{c}\right)$. In the solutions 1-(b) and 2-(b), the $\mathrm{O}(2)(\mathrm{U}(1))$ symmetry breaks down spontaneously, and they give actually the critical regions of real dimension one, parameterized by $\theta \in[0,2 \pi]$.

We take the thimbles associated with the classical vacua, 1-(a) for $\mu<\tilde{\mu}_{c}$ and 2-(b) for $\mu>\tilde{\mu}_{c}$, for our purpose. For the model parameters, we choose the values, $\kappa=1$ and $\lambda=1$, following the study in [17]. In this case, $\tilde{\mu}_{c} \simeq 0.962$. We measure the number density,

$$
n[z]=\frac{1}{L^{4}} \sum_{x} K_{0} z_{a}(x) z_{b}(x+\hat{0})\left[\delta_{a b} \sinh (\mu)-i \epsilon_{a b} \cosh (\mu)\right]
$$

as well as the residual phase factor, $\mathrm{e}^{i \phi_{z}}=\operatorname{det} V_{z} /\left|\operatorname{det} V_{z}\right|$, for various values of $\mu$ in the range $\mu \in[0,1.5] .{ }^{22}$ We consider only the lattice size $L=4$ in this work.

\footnotetext{
${ }^{22}$ In this model, the orthonormal tangent vectors at the critical point $\left\{v_{a}(x)^{\alpha}\right\}(\alpha=1, \cdots, 2 V)$ can be chosen to satisfy $C \bar{v}^{\alpha}=v^{\beta} P^{\beta \alpha}$, where $C$ is the charge conjuation operator defined by $C: z_{1}(x) \leftrightarrow z_{2}(x)$, while $P$ is a permutation operator. It then follows that $\left.\mathrm{e}^{i \phi_{z}}\right|_{z=z_{\mathrm{vac}}}=\operatorname{det} v= \pm 1$.
} 


\subsection{Thimble 1-(a) for $\mu<\tilde{\mu}_{c}$}

The algorithm given in section 3 applies straightforwardly to the thimble 1-(a) for $\mu<\tilde{\mu}_{c}$. We have generated 4,250 trajectories for each value $\mu=0.1,0.3,0.5,0.7$ and 0.9 with the parameters listed in table 1. Each trajectory is of the length $\tau_{\text {traj }}=1.0$ and obtained in the number of steps $n_{\text {step }}=20$. In solving the flow equations, the parameters are chosen as $t_{0}=-5.0$ and $n_{\text {lefs }}=100$. We have found in the course of the simulations that the scale variable $t^{\prime}$ varies within the range $[4.9,5.1]$ and $h=t^{\prime} / n_{\text {lefs }} \simeq 0.05$ most of the time, and the solutions satisfy the bounds, $|\operatorname{Im} S[z]| \lesssim 1.0 \times 10^{-4}$ and $\left\|\bar{\partial} \bar{S}-V^{\alpha} \kappa^{\alpha} e^{\alpha}\right\|^{2} / 2 V \leq 1.0 \times 10^{-4}$. In solving the constraint in the molecular dynamics, the fixed-point method converges with the iteration numbers $l \lesssim 4$ for the step size $\Delta \tau=\tau_{\text {traj }} / n_{\text {step }}=0.05$ and the bound $\epsilon^{\prime}=1.0 \times 10^{-3} . \Delta H$ turns out to be rather small, and the acceptance rates are $\simeq 0.99$ on average. The integrated auto-correlation times are estimated as $\tau_{\text {int }} \simeq 2$ for $\operatorname{Re} S[z]$ and $\tau_{\text {int }} \simeq 3$ for $\phi_{z}$ for all the given values of $\mu$. In fig. 2, Monte Carlo histories of $\operatorname{Re} S[z]$ are shown for $\mu=0.5$ and 0.9 . (As for $\operatorname{Im} S[z]$, its absolute value is kept less than $1.0 \times 10^{-4}$ in all trajectories.) In fig. 3, Monte Carlo histories of the residual phase $\phi_{z}$ are shown for $\mu=0.5$ and 0.9 .

Table 1. Simulation parameters for the thimble 1-(a) $\left(\mu<\tilde{\mu}_{c}\right)$

\begin{tabular}{|l|l|l|}
\hline & Parameters & Resulting conditions \\
\hline Thimble & $t_{0}=-5.0$ & $\left|\operatorname{Re}\left(S\left[z\left(t_{0}\right)\right]-S\left[z_{\text {vac }}\right]\right)\right| \lesssim 1.0$ \\
(Solving flow eqs.) & $n_{\text {lefs }}=100$ & $|\operatorname{Im} S[z]| \lesssim 1.0 \times 10^{-4}$ \\
& $h=t^{\prime} / n_{\text {lefs }} \simeq 0.05$ & $\left\|\bar{\partial} \bar{S}-V^{\alpha} \kappa^{\alpha} e^{\alpha}\right\|^{2} / 2 V \leq 1.0 \times 10^{-4}$ \\
\hline Molecular Dynamics & $\tau_{\text {traj }}=1.0$ & scale variable range $: t^{\prime} \in[4.9,5.1]$ \\
(Solving constraint) & $n_{\text {step }}=20$ & $\Delta H \lesssim 0.1$ \\
& $\Delta \tau=0.05$ & acceptance rate $\simeq 0.99$ \\
& $\epsilon^{\prime}=1.0 \times 10^{-3}$ & number of iterations : $l \lesssim 4$ \\
\hline Auto-corr. time & & $\tau_{\text {int }} \simeq 2$ for $\operatorname{Re} S[z]$ \\
& & $\tau_{\text {int }} \simeq 3$ for $\phi_{z}$ \\
\hline
\end{tabular}

We have made measurements of $n[z]$ and $\mathrm{e}^{i \phi_{z}}$ using 300 trajectories out of 4,250 with separations of 10 , discarding the first 1,250 for thermalization. The numerical results of $\left\langle\mathrm{e}^{i \phi_{z}}\right\rangle_{\mathcal{J}_{\text {vac }}}^{\prime}$, listed in table 2, suggest that the reweighting would work for all the given values of $\mu\left(<\tilde{\mu}_{c}\right)$. The result of $\langle n[z]\rangle_{\mathcal{J}_{\mathrm{vac}}}$, based on the formula eq. (3.29), is shown in fig. 4. The errors are those estimated by the jack-knife method. 

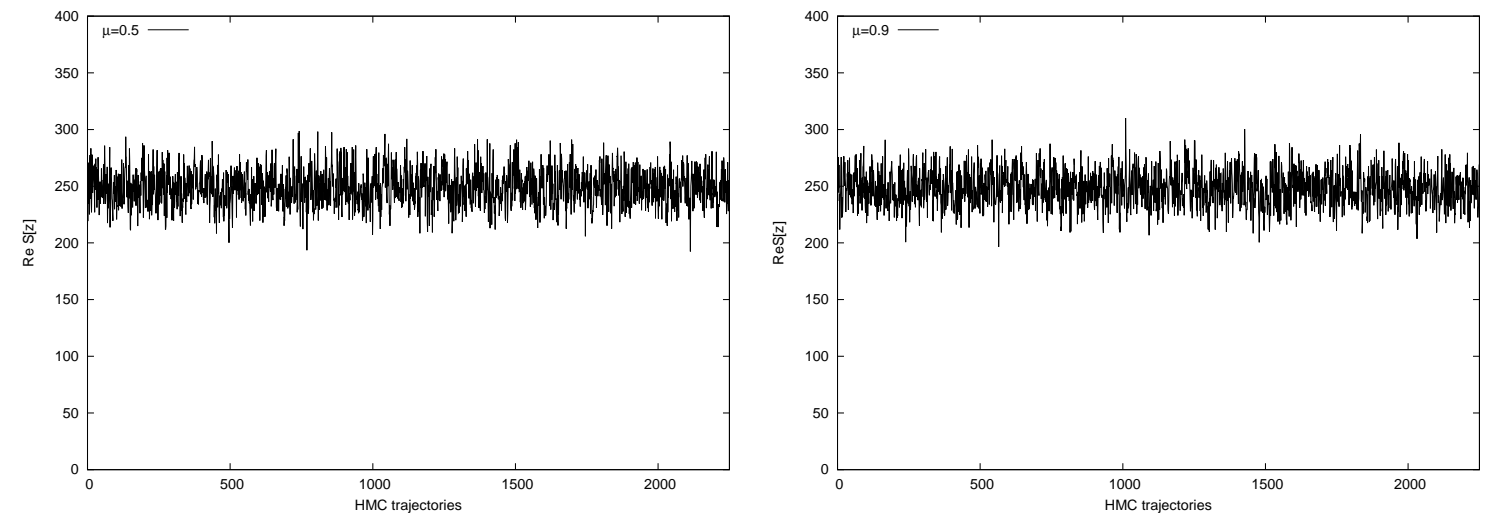

Figure 2. Monte Carlo histories of $\operatorname{Re} S[z]$ for $\mu=0.5$ and $0.9(\kappa=1.0, \lambda=1.0, L=4)$. In the course of the MC updates, the absolute values of $\operatorname{Im} S[z]$ were kept less than $1.0 \times 10^{-4}$.
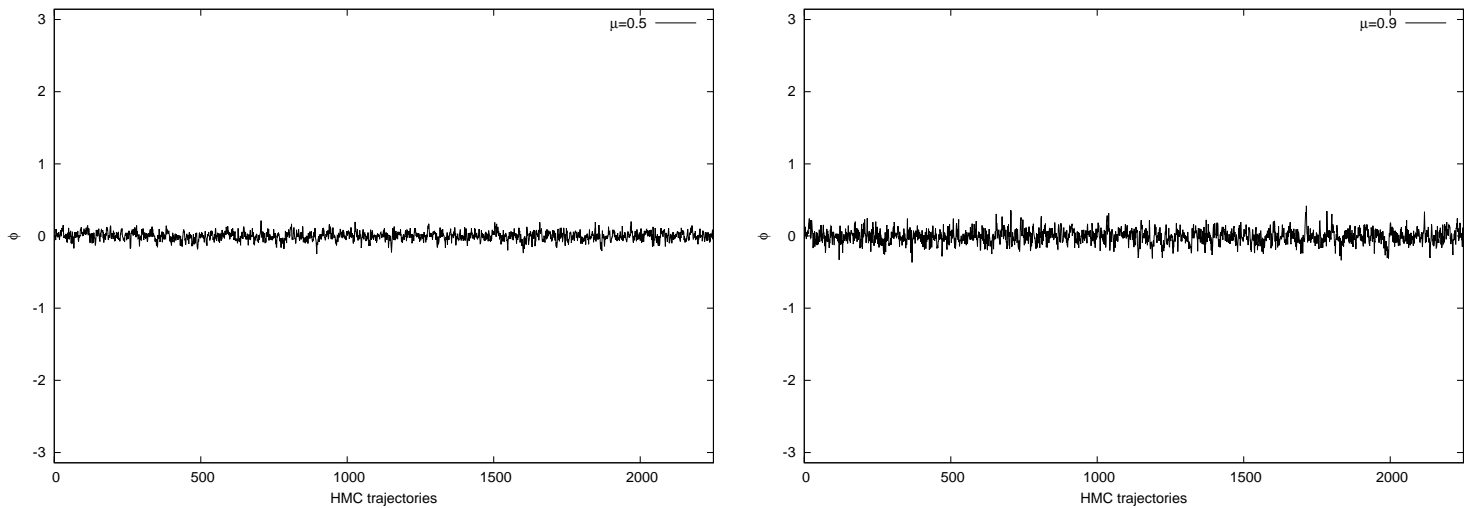

Figure 3. Monte Carlo histories of $\phi_{z}$ for $\mu=0.5$ and $0.9(\kappa=1.0, \lambda=1.0, L=4)$.

Table 2. Averages of the residual phase factors. The errors are statistical ones.

\begin{tabular}{|c|c|}
\hline$\mu$ & $\left\langle\mathrm{e}^{i \phi_{z}}\right\rangle_{\mathcal{J}_{\text {vac }}}^{\prime}$ \\
\hline 0.1 & $(9.99 \mathrm{e}-01,-1.15 \mathrm{e}-03) \pm(5.7 \mathrm{e}-02,7.4 \mathrm{e}-04)$ \\
0.3 & $(9.99 \mathrm{e}-01,-1.03 \mathrm{e}-03) \pm(5.7 \mathrm{e}-02,2.1 \mathrm{e}-03)$ \\
0.5 & $(9.98 \mathrm{e}-01,-2.68 \mathrm{e}-03) \pm(5.7 \mathrm{e}-02,3.3 \mathrm{e}-03)$ \\
0.7 & $(9.97 \mathrm{e}-01,5.24 \mathrm{e}-04) \pm(5.7 \mathrm{e}-02,4.3 \mathrm{e}-03)$ \\
0.9 & $(9.94 \mathrm{e}-01,-7.40 \mathrm{e}-03) \pm(5.7 \mathrm{e}-02,5.9 \mathrm{e}-03)$ \\
\hline
\end{tabular}




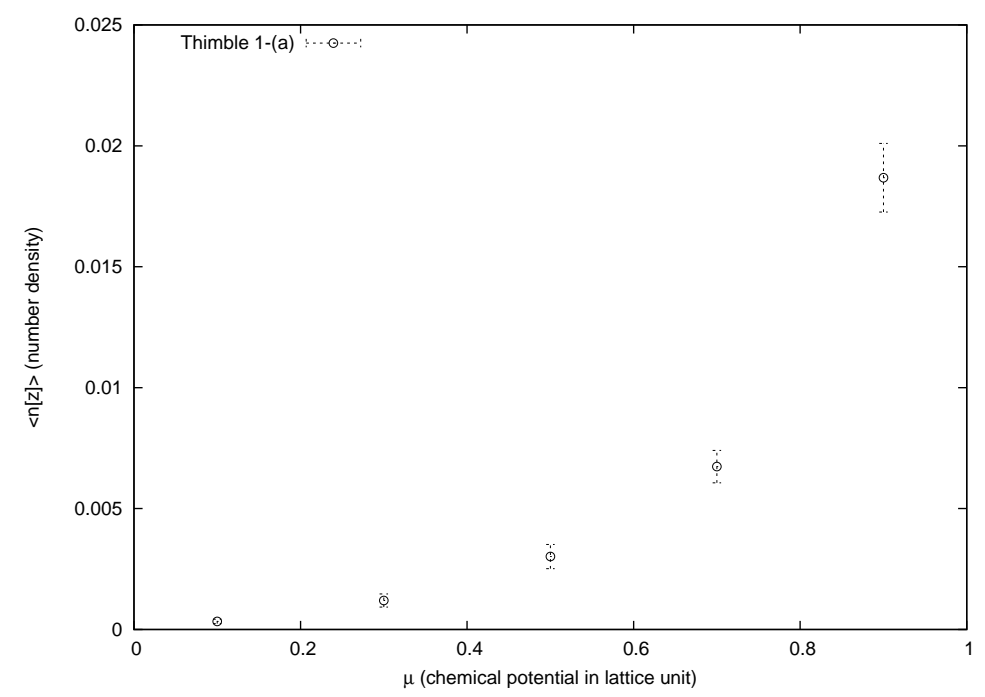

Figure 4. The expectation values of $n[z]$ evaluated on the thimble $1-(\mathrm{a})\left(\mu<\tilde{\mu}_{c}\right)$. The errors are those estimated by the jack-knife method.

\subsection{Thimble 2-(b) for $\mu>\tilde{\mu}_{c}$}

On the other hand, when applied to the thimble 2-(b) for $\mu>\tilde{\mu}_{c}$, the algorithm in section 3 requires a few modifications in the parametrization of the thimble. This is because the thimble of 2-(b) has the critical region of dimension one and there appears a zero mode $\kappa^{0}(=0)$ which corresponds to the degrees of freedom in the parameter $\theta$ (i.e. the zeromomentum modes of the Nambu-Goldstone boson $\pi$ ). In fact, the asymptotic solution to the flow equation in this case is given by

$$
z_{a}(x ; t) \simeq R_{a b}(\theta)\left\{\delta_{b 1} \phi_{0}+\sum_{\beta=1}^{2 V-1} v_{b}(x)^{\beta} \exp \left(\kappa^{\beta} t\right) e^{\beta}\right\} \quad(t \ll 0),
$$

where the direction vector $e^{\beta}$ is $(2 V-1)$-dimensional and normalized as $\sum_{\beta=1}^{2 V-1} e^{\beta} e^{\beta}=$ $(2 V-1)$, and $R(\theta) \in \mathrm{O}(2): R_{11}=R_{22}=\cos \theta$ and $R_{21}=-R_{12}=\sin \theta \cdot{ }^{23}$ As for the variation $\delta z_{a}(x ; t)$, it follows that

$$
\delta z_{a}(x ; t)=V_{a}(x ; t)^{0}\left(\phi_{0} \sqrt{V} \delta \theta\right)+\sum_{\beta=1}^{2 V-1} V_{b}(x ; t)^{\beta}\left(\delta e^{\beta}+\kappa^{\beta} e^{\beta} \delta t\right) .
$$

We regard $\theta$ as a dynamical variable in the molecular dynamics. According to the equations of motion eqs. (3.4) and (3.5), it obeys $\phi_{0} \sqrt{V} \dot{\theta}=(w)^{0}$ and $(\dot{w})^{0}=0$ because $\kappa^{0}=0$.

Furthermore, when $\mu$ is close to $\tilde{\mu}_{c}\left(\mu \gtrsim \tilde{\mu}_{c}\right)$, the lowest lying non-zero mode with $\kappa^{1}=2 \lambda_{0} \phi_{0}^{2}$ and $v_{a}(x)^{1}=\delta_{a 1} / \sqrt{V}$ (i.e. the zero-momentum mode of the scalar boson $\sigma$ )

\footnotetext{
${ }^{23}$ See appendix for the expressions of $v_{a}(x)^{\beta}$ and $\kappa^{\beta}$ for $\beta=0,1, \cdots, 2 V-1$.
} 
tends to be very light ${ }^{24}$ and, due to critical fluctuations, ${ }^{25}$ the component $e^{1}$ can dominate the direction vector $e^{\beta}$. This implies that the factor $\exp \left(\kappa^{1} t\right) e^{1}$ in the asymptotic solution eq. (4.7) is not a small number unless $t$ (or $t_{0}$ ) assumes a very large negative value, and this can invalidate the linear approximation to the flow equations. ${ }^{26}$ To improve this situation, we note that for the global flow mode $z_{a}(x ; t)=z_{a}(t)$, the flow equation reads

$$
\begin{aligned}
\frac{d}{d t} z_{a}(t) & =\left.\bar{\partial}_{a x} \bar{S}[\bar{z}]\right|_{z_{a}(x ; t)=z_{a}(t)} \\
& =\lambda_{0}\left(\bar{z}_{b}(t) \bar{z}_{b}(t)-\phi_{0}^{2}\right) \bar{z}_{a}(t),
\end{aligned}
$$

and the exact solution to the non-linear flow equation is obtained explicitly as

$$
z_{a}(t)=R_{a b}(\theta) \delta_{b 1} \frac{\phi_{0}}{\sqrt{1-\frac{2}{\sqrt{V} \phi_{0}} e^{1} \exp \left(\kappa^{1} t\right)}} .
$$

Here the allowed range of $t$ is $\left[-\infty, t^{*}\right]$ where $t^{*}=\ln \left(\sqrt{V} \phi_{0} / 2 e^{1}\right) / \kappa^{1}$, and $e^{1}$ takes a value in the range $\left[-\infty, e^{1^{*}}\right]$ where $e^{1^{*}}=\sqrt{V} \phi_{0} \exp \left(-\kappa^{1} t_{0}\right) / 2$ for $t=t_{0}(\ll 0)$ fixed. This leads us to adopt the following asymptotic form for $t \ll 0$,

$$
z_{a}(x ; t) \simeq R_{a b}(\theta)\left\{\delta_{b 1} \frac{\phi_{0}}{\sqrt{1-\frac{2}{\sqrt{V} \phi_{0}} e^{1} \exp \left(\kappa^{1} t\right)}}+\sum_{\beta=2}^{2 V-1} v_{b}(x)^{\beta} \exp \left(\kappa^{\beta} t\right) e^{\beta}\right\},
$$

where the direction vector $e^{\beta}$ is normalized as $\sum_{\beta=2}^{2 V-1} e^{\beta} e^{\beta}=2 V-2$ excluding $e^{1}$. Accordingly, for the tangent vectors, we adopt the following asymptotic forms for $t \ll 0$,

$$
\begin{aligned}
& V_{a}(x ; t)^{0} \simeq R_{a b}(\theta) v_{b}(x)^{0} \frac{1}{\sqrt{1-\frac{2}{\sqrt{V} \phi_{0}} e^{1} \exp \left(\kappa^{1} t\right)}}, \\
& V_{a}(x ; t)^{1} \simeq R_{a b}(\theta) v_{b}(x)^{1} \frac{\exp \left(\kappa^{1} t\right)}{\left(1-\frac{2}{\sqrt{V} \phi_{0}} e^{1} \exp \left(\kappa^{1} t\right)\right)^{3 / 2}}, \\
& V_{a}(x ; t)^{\beta} \simeq R_{a b}(\theta) v_{b}(x)^{\beta} \exp \left(\kappa^{\beta} t\right) \quad(\beta=2, \cdots, 2 V-1),
\end{aligned}
$$

where $v_{a}(x)^{0}=\delta_{a 2} / \sqrt{V} \cdot{ }^{27}$

\footnotetext{
${ }^{24}$ Here we assume the lattice size $L$ is relatively small. For a large $L$, there also appear light non-zero momentum modes of the scalar and Nambu-Goldstone bosons.

${ }^{25}$ The critical point of the second-order phase transition in this system is $\mu_{c} \simeq 1.15\left(\gtrsim \tilde{\mu}_{c}\right)$ for $\kappa=1, \lambda=1$, as shown in $[17,18]$.

${ }^{26}$ One should also note the fact that the truncation errors in the linear approximation are of order $\lambda_{0} z^{3}$ for the critical points 1-(a) $\left(\mu<\tilde{\mu}_{c}\right)$, but of order $\lambda_{0} \phi_{0}\left(z-\phi_{0}\right)^{2}$ for the critical point 2-(b) $\left(\mu>\tilde{\mu}_{c}\right)$. For the latter case, it is relatively hard to reach the asymptotic region.

${ }^{27}$ The tangent vectors $V_{a}(x ; t)^{0}$ and $V_{a}(x ; t)^{1}$ in $(4.12)$ and (4.13), respectively are indeed the exact solutions to the flow equations with the global flow mode $z_{a}(x ; t)=z_{a}(t)$ :

$$
\begin{aligned}
\frac{d}{d t} V_{a}(x ; t)^{\beta} & =\left.\bar{\partial}_{a x} \bar{\partial}_{b y} \bar{S}[\bar{z}]\right|_{z_{a}(x ; t)=z_{a}(t)} \bar{V}_{b}(y ; t)^{\beta} \\
& =K_{0} \Delta_{a b} \bar{V}_{b}(x ; t)^{\beta}+\lambda_{0}\left(\bar{z}_{b}(t) \bar{z}_{b}(t)-\phi_{0}^{2}\right) \bar{V}_{a}(x ; t)^{\beta}+2 \lambda_{0} \bar{z}_{a}(t) \bar{z}_{b}(t) \bar{V}_{b}(x ; t)^{\beta},
\end{aligned}
$$

where $\Delta_{a b}=\left\{\nabla_{k} \nabla_{k}^{*}+\cosh (\mu) \nabla_{0} \nabla_{0}^{*}\right\} \delta_{a b}-i \sinh (\mu)\left(\nabla_{0}+\nabla_{0}^{*}\right) \epsilon_{a b}$. The similar exact solutions for $V_{a}(x ; t)^{\beta}$ $(\beta=2, \cdots, 2 V-1)$ can be worked out, but the results turns out to be involved. We therefore adopt the simpler solutions to the linearized flow equation as in (4.14), although the consistency in the linear approximation is lost.
} 
Using the algorithm with the above modifications, we have generated 11, 250 trajectories for each value $\mu=1.0,1.1,1.2,1.3$, and 1.5 with the parameters listed in table 3 . In this case, each trajectory has the length $\tau_{\text {traj }}=0.3$ and obtained in the number of steps $n_{\text {step }}=30(\mu=1.0,1.1)$ and $10(\mu=1.2,1.3,1.5)$. In solving the flow equations, the parameters are chosen as $t_{0}=-3.0$ and $n_{\text {lefs }}=100$. In the course of the updates, we have found that $t^{\prime} \in[2.5,3.5]$ and $h=t^{\prime} / n_{\text {lefs }} \simeq 0.03$ most of the time, and the solutions satisfy the bounds, $\left|\operatorname{Im}\left(S[z]-S\left[z_{\text {vac }}\right]\right)\right| \lesssim 5.0 \times 10^{-2}$ and $\left\|\bar{\partial} \bar{S}-V^{\alpha} \kappa^{\alpha} e^{\alpha}\right\|^{2} / 2 V \lesssim 3.0 \times 10^{-2}$. In solving the constraint in the molecular dynamics, the fixed-point method converges with iteration numbers $l \leq 6(\mu=1.0), 14(\mu=1.1), 4(\mu=1.2,1.3,1.5)$ for the step sizes $\Delta \tau=$ $\tau_{\text {traj }} / n_{\text {step }}=0.01(\mu=1.0,1.1), 0.03(\mu=1.2,1.3,1.5)$ and the bound $\epsilon^{\prime}=\sqrt{10} \times 10^{-3}$. It has occurred twice for $\mu=1.0$ and once for $\mu=1.1$ that the fixed point method failed to converge. For such trajectories, the momenta have been re-refreshed and the molecular dynamics has been re-started. ${ }^{28}$

Table 3. Simulation parameters for the thimble 2-(b) $\left(\mu>\tilde{\mu}_{c}\right)$

\begin{tabular}{|l|l|l|}
\hline & Parameters & Resulting conditions \\
\hline Thimble & $t_{0}=-3.0$ & $\left|\operatorname{Re}\left(S\left[z\left(t_{0}\right)\right]-S\left[z_{\text {vac }}\right]\right)\right| \lesssim 2.0 \times 10^{1}$ \\
& $n_{\text {lefs }}=100$ & $\left|\operatorname{Im}\left(S[z]-S\left[z_{\text {vac }}\right]\right)\right| \lesssim .0 \times 10^{-2}$ \\
& $h=t^{\prime} / n_{\text {lefs }} \simeq 0.03$ & $\left\|\bar{\partial} \bar{S}-V^{\alpha} \kappa^{\alpha} e^{\alpha}\right\|^{2} / 2 V \leq 3.0 \times 10^{-2}$ \\
\hline MD & $\tau_{\text {traj }}=0.3$ & $t^{\prime} \in[2.5,3.5]$ \\
& $n_{\text {step }}=10,30(\mu=1.0,1.1)$ & $\Delta H \lesssim 0.05$ \\
& $\Delta \tau=0.03,0.01(\mu=1.0,1.1)$ & Acceptance rate $\simeq 0.99$ \\
& $\epsilon^{\prime}=\sqrt{10} \times 10^{-3}$ & $l \lesssim 4,6(\mu=1.0), 14(\mu=1.1)$ \\
\hline Auto-corr. time & $($ for $\operatorname{Re} S[z])$ & $\tau_{\text {int }} \simeq 10,14(\mu=1.0,1.1)$ \\
& $\left(\right.$ for $\left.\phi_{z}\right)$ & $\tau_{\text {int }} \simeq 15,14(\mu=1.0), 28(\mu=1.1)$ \\
\hline
\end{tabular}

We have made measurements of $n[z]$ and $\mathrm{e}^{i \phi_{z}}$ using 1,000 trajectories out of 11,250 with separations of 10, discarding the first 1,250 for thermalization. The numerical result of $\left\langle\mathrm{e}^{i \phi_{z}}\right\rangle_{\mathcal{J}_{\mathrm{vac}}}^{\prime}$, listed in table 4 , suggests again that the reweighting would work for all the given values of $\mu\left(>\tilde{\mu}_{c}\right)$. The result of $\langle n[z]\rangle_{\mathcal{J}_{\text {vac }}}$, based on the formula eq. (3.29), is shown in fig. 5 . The errors are those estimated by the jack-knife method.

\footnotetext{
28 As far as we understand, these failures have occurred due to our implementation of the algorithm. The asymptotic solution is in the form of the "polar decomposition" as $z_{a} \simeq R_{a 1}(\theta) \rho$, where $\rho=\phi_{0} / \sqrt{1-2 e^{1} \mathrm{e}^{\kappa^{1} t} / \phi_{0} \sqrt{V}}$. The factor $\rho$ can be rather small for $\mu \gtrsim \tilde{\mu}_{c}$, and it can even be negative in the updates with a finite step size. In such a case, one needs to do a coordinate transformation such as $(\rho, \theta) \rightarrow(-\rho, \theta+\pi)$. This procedure is in fact neglected in our implementation, and we have instead managed with the reduced step size $\Delta \tau=0.01(\mu=1.0,1.1)$.
} 
Table 4. Averages of the residual phase factor. The errors are statistical ones.

\begin{tabular}{|c|c|}
\hline$\mu$ & $\left\langle\mathrm{e}^{i \phi_{z}}\right\rangle_{\mathcal{J}_{\text {vac }}}^{\prime}$ \\
\hline 1.0 & $(9.94 \mathrm{e}-01,-8.77 \mathrm{e}-03) \pm(3.1 \mathrm{e}-02,3.1 \mathrm{e}-03)$ \\
1.1 & $(9.94 \mathrm{e}-01,-3.21 \mathrm{e}-03) \pm(3.1 \mathrm{e}-02,3.4 \mathrm{e}-03)$ \\
1.2 & $(9.95 \mathrm{e}-01,-8.25 \mathrm{e}-04) \pm(3.1 \mathrm{e}-02,3.0 \mathrm{e}-03)$ \\
1.3 & $(9.97 \mathrm{e}-01,-3.08 \mathrm{e}-03) \pm(3.1 \mathrm{e}-02,2.2 \mathrm{e}-03)$ \\
1.5 & $(9.99 \mathrm{e}-01,-1.06 \mathrm{e}-03) \pm(3.1 \mathrm{e}-02,1.0 \mathrm{e}-03)$ \\
\hline
\end{tabular}

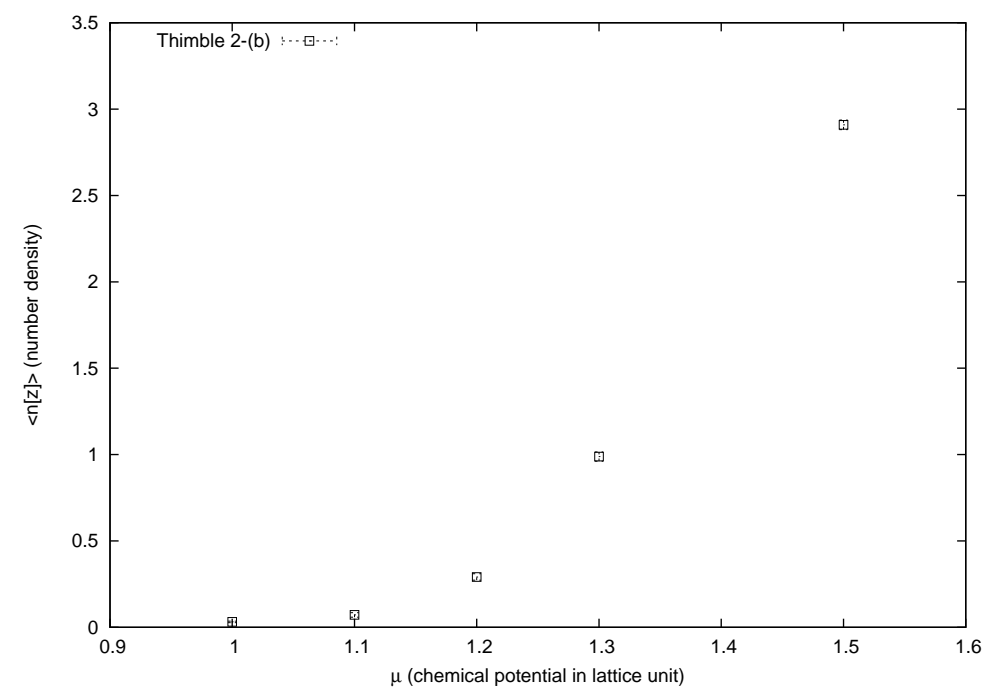

Figure 5. The expectation values of $n[z]$ evaluated on the thimble 2-(b) $\left(\mu>\tilde{\mu}_{c}\right)$. The errors are those estimated by the jack-knife method.

\subsection{A comparison to the results of the complex Langevin simulations}

In fig. 6, the results of $\langle n[z]\rangle_{\mathcal{J}_{\text {vac }}}$ on the two thimbles, 1-(a) for $\mu<\tilde{\mu}_{c}$ and 2-(b) for $\mu>\tilde{\mu}_{c}$, are shown together. The numerical data are summerized in table 5 .

It is instructive to compare our numerical results with those obtained by the complex Langevin equation[17] and the dual variable method[32-34]. We have reproduced the expectation values of $n[z]$ through the complex Langevin simulations with the step size $\epsilon=$ $5.0 \times 10^{-5}$, samping 10,000 configurations with separation of 500 out of $5.0 \times 10^{6}$ timesteps. These results are shown in fig. 7 with our results by the hybrid Monte Carlo. The two sets of the results are in agreement within the statistical errors, except for $\mu=0.7,1.2,1.3$, and overall, they are consistent with each other. 


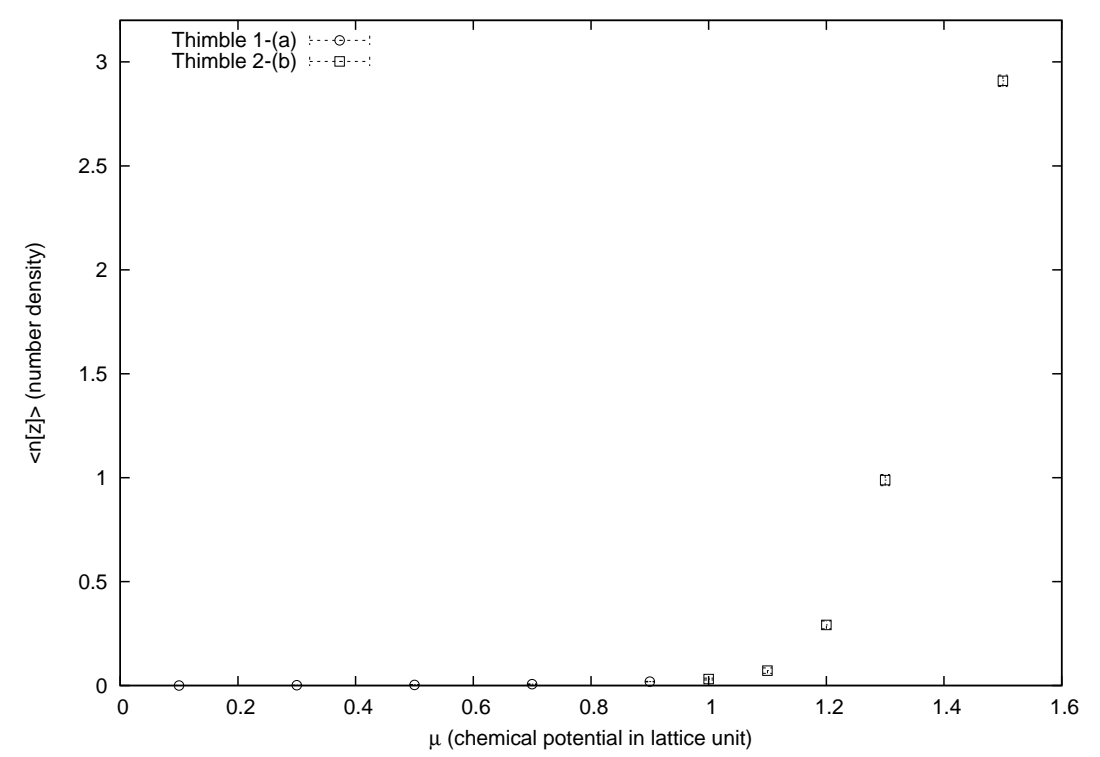

Figure 6. The expectation values of $n[z]$ evaluated on both thimbles, 1-(a) for $\mu<\tilde{\mu}_{c}$ and 2-(b) for $\mu>\tilde{\mu}_{c}$. The errors are those estimated by the jack-knife method.

Table 5. Numerical data of the expectation values of $n[z]$

\begin{tabular}{|c|cl|c|c|}
\hline$\mu$ & $\operatorname{Re}\langle n[z]\rangle_{\mathcal{J}_{\text {vac }}}$ & (j.-k. error $)$ & $\operatorname{Re}\left\langle\mathrm{e}^{i \phi_{z}} n[z]\right\rangle_{\mathcal{J}_{\text {vac }}}^{\prime}$ & $\operatorname{Re}\langle n[z]\rangle_{\mathcal{J}_{\text {vac }}}$ \\
\hline 0.1 & $3.34 \mathrm{e}-04$ & $(9.2 \mathrm{e}-05)$ & $3.35 \mathrm{e}-04$ & $2.15 \mathrm{e}-04$ \\
0.3 & $1.20 \mathrm{e}-03$ & $(2.7 \mathrm{e}-04)$ & $1.19 \mathrm{e}-03$ & $8.56 \mathrm{e}-04$ \\
0.5 & $3.02 \mathrm{e}-03$ & $(5.0 \mathrm{e}-04)$ & $3.01 \mathrm{e}-03$ & $2.44 \mathrm{e}-03$ \\
0.7 & $6.74 \mathrm{e}-03$ & $(6.7 \mathrm{e}-04)$ & $6.71 \mathrm{e}-03$ & $5.91 \mathrm{e}-03$ \\
0.9 & $1.89 \mathrm{e}-02$ & $(1.4 \mathrm{e}-03)$ & $1.85 \mathrm{e}-02$ & $1.73 \mathrm{e}-02$ \\
1.0 & $3.14 \mathrm{e}-02$ & $(4.3 \mathrm{e}-03)$ & $3.12 \mathrm{e}-02$ & $3.00 \mathrm{e}-02$ \\
1.1 & $7.17 \mathrm{e}-02$ & $(1.3 \mathrm{e}-02)$ & $7.12 \mathrm{e}-02$ & $7.01 \mathrm{e}-02$ \\
1.2 & $2.92 \mathrm{e}-01$ & $(1.8 \mathrm{e}-02)$ & $2.90 \mathrm{e}-01$ & $2.90 \mathrm{e}-01$ \\
1.3 & $9.88 \mathrm{e}-01$ & $(2.6 \mathrm{e}-02)$ & $9.85 \mathrm{e}-01$ & $9.87 \mathrm{e}-01$ \\
1.5 & $2.91 \mathrm{e}-00$ & $(2.7 \mathrm{e}-02)$ & $2.90 \mathrm{e}-00$ & $2.90 \mathrm{e}-00$ \\
\hline
\end{tabular}



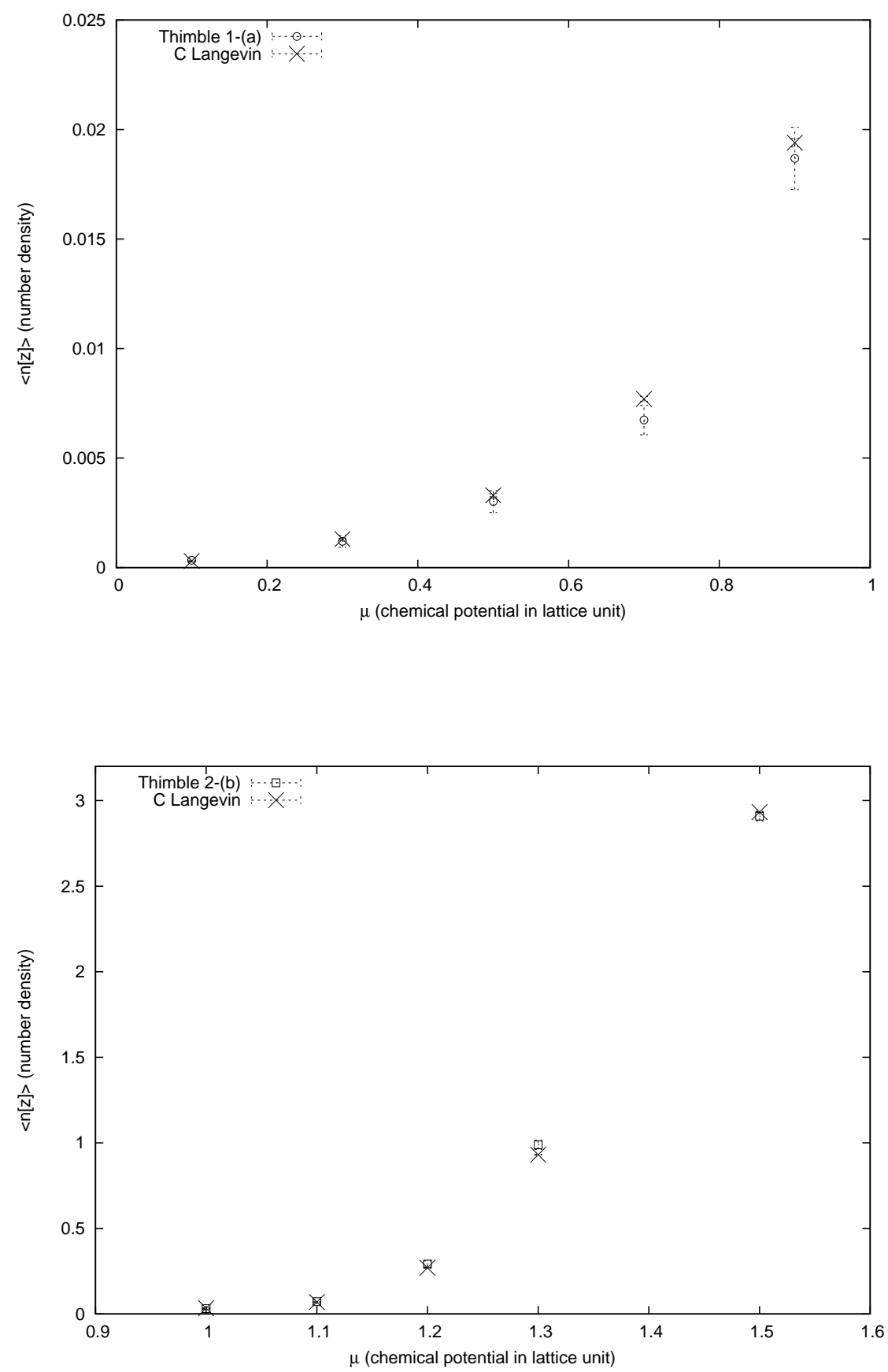

Figure 7. The expectation values of $n[z]$ evaluated by the complex Langevin simulations in comparison with those by the hybrid Monte Carlo. 


\section{Summary and Discussion}

In this article, we have introduced the hybrid Monte Carlo algorithm which is applicable to lattice models defined on Lefschetz thimbles. We have tested the algorithm in the $\lambda \phi^{4}$ model with the couplings $\kappa=1.0$ and $\lambda=1.0$, the chemical potential $\mu \in[0.0,1.5]\left(\tilde{\mu}_{c} \simeq 0.962\right)$, and the lattice size $L=4$. We have found that the algorithm can indeed be applied to the thimbles associated with the classical vacua and can produce the expectation values of the number density, $n[z]$, which are consistent with those obtained by the other methods[17, 32 34]. In particular, we have shown that the residual sign factors, $\mathrm{e}^{i \phi_{z}}=\operatorname{det} V_{z} /\left|\operatorname{det} V_{z}\right|$, average to not less than 0.99 , and can be safely included by reweighting for all the values of $\mu$ studied within the range $[0.0,1.5]$. This result is in sharp contrast to the fact that the phase-quenched Monte Carlo method based on the real part of the action, $\operatorname{Re} S[x]$, fails because the averages of the exponent of the imaginary part of the action, $\left\langle\mathrm{e}^{-i \operatorname{Im} S[x]}\right\rangle$, get vanishingly small for $\mu \gtrsim \tilde{\mu}_{c}$ even at the lattice size $L=4[17,36]$.

As a next step, we certainly need to examine in detail the systematic errors in the hybrid Monte Carlo method, in particular, those in defining the asymptotic regions of the thimbles and in neglecting the possible contributions of the other thimbles. In this respect, it is somewhat surprising to observe the agreement of the two set of the results shown in fig. 7 , in particular, for the values of $\mu$ close to $\tilde{\mu}_{c}$, because we expect that the contributions of the other thimbles such as that associated with 2-(a) would become important there. We should also extend the study of the residual sign problem in the $\lambda \phi^{4}$ model to larger lattice sizes. The numerical cost per trajectory in the algorithm scales as $\mathcal{O}\left(V^{2} n_{\text {lefs }} \times n_{\text {step }}\right)$ in the computation of the tangent vectors $\left\{V_{z}^{\alpha}\right\}(\alpha=1, \cdots, 2 V)$ and as $\mathcal{O}\left(V^{3} \times n_{\text {step }}\right)$ in the computation of the inverse $V_{z}^{-1}$ and the determinant $\operatorname{det} V_{z}$, and it would be challenging for the large lattice sizes. ${ }^{29}$ A study on these points will be reported in a forth coming paper.

For a future study, it would be interesting to apply the hybrid Monte Carlo method to lattice QCD at finite density. Results in this approach, even at small lattices, would serve as a cross check of the results obtained recently in the complex Langevin approach[29].

\section{Acknowledgments}

The authors would like to thank Koji Hukushima for enlightening conversations. H.F., Y.K., and T.S. are grateful to J. Bloch and F. Bruckmann for their hospitality at the International Workshop on the Sign Problem in QCD and Beyond, and they are also grateful to L. Scorzato for valuable discussions. The authors would also like to thank G. Aarts for his comments and for sending us the numerical data of his results. This work is supported in part by JSPS KAKENHI Grant Numbers 24540255 (H.F.), 25287049 (M.K.), 24540253 (Y.K.). D.H. and S.K. are supported in part by JSPS Research Fellowship for Young Scientists.

\footnotetext{
${ }^{29}$ For this, it would be essential to execute the computation of the tangent vectors in parallel using GPUs (cluster).
} 


\section{A Tangent vectors at the critical point of the thimble 2-(b)}

In this appendix, we give the explicit formulae of the tangent vectors $\left\{v_{a}(x)^{\alpha}\right\}$ at the critical point of the thimble 2-(b). Let us label the tangent vectors by the set of indices $\alpha=(k, \delta)$, where $k=\left(k_{0}, k_{1}, k_{2}, k_{3}\right)\left(k_{0}=0, \cdots, L / 2 ; k_{i}=0, \cdots, L-1(i=1,2,3)\right)$ and $\delta=1,2,3,4(\delta=1,3$ for $k=(0,0,0,0)$ and $(L / 2,0,0,0))$. Then $\kappa(k, \delta)$ and $v_{a}(x ; k, \delta)$ are given as follows:

1. $\kappa(k, 1)=\Delta_{\sigma} c^{2}-\Delta_{\pi} s^{2}+2 S c s:$

$$
v_{a}(x ; k, 1)=(+1) \sqrt{\frac{1}{L}}\left(\begin{array}{c}
c \cos \left(\frac{2 \pi}{L} k_{0} x_{0}\right) \\
-i s \sin \left(\frac{2 \pi}{L} k_{0} x_{0}\right)
\end{array}\right) \prod_{i=1}^{3} T_{i}\left(x_{i} ; k_{i}\right)
$$

2. $\kappa(k, 2)=\Delta_{\sigma} c^{2}-\Delta_{\pi} s^{2}+2 S c s:$

$$
v_{a}(x ; k, 2)=(-i) \sqrt{\frac{1}{L}}\left(\begin{array}{c}
i c \sin \left(\frac{2 \pi}{L} k_{0} x_{0}\right) \\
-s \cos \left(\frac{2 \pi}{L} k_{0} x_{0}\right)
\end{array}\right) \prod_{i=1}^{3} T_{i}\left(x_{i} ; k_{i}\right)
$$

3. $\kappa(k, 3)=-\Delta_{\sigma} s^{2}+\Delta_{\pi} c^{2}+2 S c s$ :

$$
v_{a}(x ; k, 3)=(+1) \sqrt{\frac{1}{L}}\left(\begin{array}{c}
i s \sin \left(\frac{2 \pi}{L} k_{0} x_{0}\right) \\
c \cos \left(\frac{2 \pi}{L} k_{0} x_{0}\right)
\end{array}\right) \prod_{i=1}^{3} T_{i}\left(x_{i} ; k_{i}\right)
$$

4. $\kappa(k, 4)=-\Delta_{\sigma} s^{2}+\Delta_{\pi} c^{2}+2 S c s$ :

$$
v_{a}(x ; k, 4)=(-i) \sqrt{\frac{1}{L}}\left(\begin{array}{c}
s \cos \left(\frac{2 \pi}{L} k_{0} x_{0}\right) \\
i c \sin \left(\frac{2 \pi}{L} k_{0} x_{0}\right)
\end{array}\right) \prod_{i=1}^{3} T_{i}\left(x_{i} ; k_{i}\right)
$$

where

$$
T_{i}\left(x_{i} ; k_{i}\right) \equiv \begin{cases}\sqrt{\frac{1}{L}} & \left(k_{i}=0\right) \\ \sqrt{\frac{2}{L}} \cos \left(\frac{2 \pi}{L} k_{i} x_{i}\right) & \left(k_{i}=1, \cdots, L / 2-1\right) \\ \sqrt{\frac{1}{L}}(-1)^{x_{i}} & \left(k_{i}=L / 2\right) \\ \sqrt{\frac{2}{L}} \sin \left(\frac{2 \pi}{L} k_{i} x_{i}\right) & \left(k_{i}=L / 2+1, \cdots, L-1\right)\end{cases}
$$

$(i=1,2,3)$,

$$
\begin{aligned}
\Delta_{\pi}(k) & =\left(2 K_{0}\right)\left[\sum_{i=1}^{3}\left(1-\cos \left(\frac{2 \pi}{L} k_{i}\right)\right)+\left(1-\cos \left(\frac{2 \pi}{L} k_{0}\right)\right) \cosh (\mu)\right], \\
\Delta_{\sigma}(k) & =\Delta_{\pi}(k)+2 \lambda_{0}\langle\phi\rangle^{2} \\
S(k) & =2 K_{0} \sin \left(\frac{2 \pi}{L} k_{0}\right) \sinh (\mu),
\end{aligned}
$$


and

$$
\begin{aligned}
c & =\left[\frac{\Delta_{\sigma}+\Delta_{\pi}}{2}+\sqrt{\left(\frac{\Delta_{\sigma}+\Delta_{\pi}}{2}\right)^{2}+S^{2}}\right] / N, \\
s & =S / N, \\
N^{2} & =\left[\frac{\Delta_{\sigma}+\Delta_{\pi}}{2}+\sqrt{\left(\frac{\Delta_{\sigma}+\Delta_{\pi}}{2}\right)^{2}+S^{2}}\right]^{2}+S^{2} .
\end{aligned}
$$

\section{References}

[1] P. de Forcrand, PoS LAT 2009, 010 (2009) [arXiv:1005.0539 [hep-lat]].

[2] S. Gupta, PoS LATTICE 2010, 007 (2010) [arXiv:1101.0109 [hep-lat]].

[3] L. Levkova, PoS LATTICE 2011, 011 (2011) [arXiv:1201.1516 [hep-lat]].

[4] S. Ejiri, Eur. Phys. J. A 49, 86 (2013) [arXiv:1306.0295 [hep-lat]].

[5] G. Aarts, PoS LATTICE 2012, 017 (2012) [arXiv:1302.3028 [hep-lat]].

[6] C. Gattringer, "New Developments for Lattice Field Theory at Non-Zero Density", the plenary talk at Lattice 2013, July 29-August 03, Mainz, Germany.

[7] G. Parisi, Phys. Lett. B 131, 393 (1983).

[8] J. R. Klauder, J. Phys. A 16, L317 (1983).

[9] J. R. Klauder, Phys. Rev. A 29, 2036 (1984).

[10] E. Witten, "Analytic Continuation Of Chern-Simons Theory," arXiv:1001.2933 [hep-th].

[11] M. Cristoforetti et al. [AuroraScience Collaboration], Phys. Rev. D 86, 074506 (2012) [arXiv:1205.3996 [hep-lat]].

[12] J. Ambjorn and S. K. Yang, Phys. Lett. B 165, 140 (1985).

[13] J. Ambjorn, M. Flensburg and C. Peterson, Nucl. Phys. B 275, 375 (1986).

[14] J. Berges, S. .Borsanyi, D. Sexty and I. -O. Stamatescu, Phys. Rev. D 75, 045007 (2007) [hep-lat/0609058].

[15] J. Berges and D. Sexty, Nucl. Phys. B 799, 306 (2008) [arXiv:0708.0779 [hep-lat]].

[16] G. Aarts and I. -O. Stamatescu, JHEP 0809, 018 (2008) [arXiv:0807.1597 [hep-lat]].

[17] G. Aarts, Phys. Rev. Lett. 102, 131601 (2009) [arXiv:0810.2089 [hep-lat]].

[18] G. Aarts, JHEP 0905, 052 (2009) [arXiv:0902.4686 [hep-lat]].

[19] G. Aarts, F. A. James, E. Seiler and I. -O. Stamatescu, Phys. Lett. B 687, 154 (2010) [arXiv:0912.0617 [hep-lat]].

[20] G. Aarts, E. Seiler and I. -O. Stamatescu, Phys. Rev. D 81, 054508 (2010) [arXiv:0912.3360 [hep-lat]].

[21] G. Aarts and F. A. James, JHEP 1008, 020 (2010) [arXiv:1005.3468 [hep-lat]].

[22] G. Aarts, F. A. James, E. Seiler and I. -O. Stamatescu, Eur. Phys. J. C 71, 1756 (2011) [arXiv:1101.3270 [hep-lat]].

[23] G. Aarts and F. A. James, JHEP 1201, 118 (2012) [arXiv:1112.4655 [hep-lat]]. 
[24] E. Seiler, D. Sexty and I. -O. Stamatescu, Phys. Lett. B 723, 213 (2013) [arXiv:1211.3709 [hep-lat]].

[25] J. M. Pawlowski and C. Zielinski, Phys. Rev. D 87, 094503 (2013) [arXiv:1302.1622 [hep-lat]].

[26] J. M. Pawlowski and C. Zielinski, Phys. Rev. D 87, 094509 (2013) [arXiv:1302.2249 [hep-lat]].

[27] G. Aarts, L. Bongiovanni, E. Seiler, D. Sexty and I. -O. Stamatescu, arXiv:1303.6425 [hep-lat].

[28] G. Aarts, P. Giudice and E. Seiler, Annals Phys. 337, 238 (2013) [arXiv:1306.3075 [hep-lat]].

[29] D. Sexty, "Simulating full QCD at nonzero density using the complex Langevin equation," arXiv:1307.7748 [hep-lat].

[30] G. Aarts, "Lefschetz thimbles and stochastic quantisation: Complex actions in the complex plane," arXiv:1308.4811 [hep-lat].

[31] P. Giudice, G. Aarts and E. Seiler, "Localised distributions in complex Langevin dynamics," arXiv:1309.3191 [hep-lat].

[32] C. Gattringer and T. Kloiber, Nucl. Phys. B 869, 56 (2013) [arXiv:1206.2954 [hep-lat]].

[33] Y. D. Mercado and C. Gattringer, Nucl. Phys. B 862, 737 (2012) [arXiv:1204.6074 [hep-lat]].

[34] C. Gattringer and A. Schmidt, Phys. Rev. D 86, 094506 (2012) [arXiv:1208.6472 [hep-lat]].

[35] M. Cristoforetti, L. Scorzato and F. Di Renzo, J. Phys. Conf. Ser. 432, 012025 (2013) [arXiv:1210.8026 [hep-lat]].

[36] M. Cristoforetti, F. Di Renzo, A. Mukherjee and L. Scorzato, arXiv:1303.7204 [hep-lat].

[37] A. Mukherjee, M. Cristoforetti and L. Scorzato, arXiv:1308.0233 [physics.comp-ph].

[38] B. Leimkuhler and S. Reich, Simulating Hamiltonian Dynamics, Cambridge University Press (2004).

[39] R.A. Horn and C.R. Johnson, Matrix Analysis, Cambridge University Press, (1985).

[40] M. Girolami and B. Calderhead, "Riemann manifold Langevin and Hamiltonian Monte Carlo methods", J. R. Statist. Soc. B (2011) 73, Part2, pp.1-37. 